\title{
Fish diversity in the cascade of reservoirs along the Paranapanema River, southeast Brazil
}

\author{
Fernando Mayer Peliciceㄹ, Valter M. Azevedo-Santos², André Luiz Henríques Esguícero ${ }^{3}$, \\ Angelo Antonio Agostinho ${ }^{4}$ and Marlene Sofia Arcifa ${ }^{3}$
}

The Paranapanema River is a major tributary of the upper Paraná river basin. Eleven hydropower dams regulate its main course, but no study has investigated fish diversity in these impoundments at the basin-scale. The present study investigated spatial patterns of richness, composition, and abundance of native (non-migratory and migratory) and non-native fishes in the cascade of reservoirs along the Paranapanema River. The study is based on data collected from 34 independent studies conducted in nine reservoirs (47 samples). The compilation recorded 161 species, being 111 native (14 migratory) and 50 nonnative. Total richness ranged between 56 and 112 species/reservoir, with a mean of 72 (49.9 non-migratory, 8.1 migratory and 14 non-native). The number of non-migratory species showed no spatial trend along the cascade system, but migratory and non-native richness increased toward downstream reaches. We also observed spatial variation in species composition along the cascade system, but some non-native fishes were widely distributed. Migratory fishes showed low relative abundance (usually $<10 \%$ ), while non-native species were common and more abundant, especially in reservoirs downstream. Our results revealed a high diversity of fishes in the cascade of impoundments, but indicated that migratory fishes are rare, while non-native species are common or dominant.

Keywords: Dam, Fish fauna, Longitudinal, Migratory fish, Non-native.

O rio Paranapanema é um dos principais afluentes da bacia do alto rio Paraná. Onze hidrelétricas regulam o seu canal principal, contudo, não existe estudo, em escala de bacia, que tenha investigado a diversidade de peixes nos represamentos. O presente estudo investigou padrões espaciais de riqueza, composição e abundância de peixes nativos (não-migradores e migradores) e não-nativos na cascata de reservatórios do rio Paranapanema. $\mathrm{O}$ estudo se baseou em dados coletados por 34 estudos independentes conduzidos em nove reservatórios (47 amostras). Registramos 161 espécies, sendo 111 nativas (14 migradoras) e 50 não-nativas. A riqueza total variou entre 56 e 112 espécies/reservatório, com média de 72 (49,9 nativas, 8,1 migradoras e 14 não-nativas). O número de espécies não-migradoras não apresentou padrão de variação ao longo do sistema em cascata, mas a riqueza de migradores e peixes não-nativos aumentou em direção aos trechos de jusante. A composição de espécies variou ao longo do gradiente longitudinal, porém algumas espécies não-nativas apresentaram ampla distribuição. Espécies migradoras apresentaram baixa abundância relativa (usualmente $<10 \%$ ), enquanto que as não-nativas foram comuns e abundantes, especialmente em reservatórios de jusante. Os resultados revelaram alta diversidade de peixes na cascata de reservatórios, mas indicaram que peixes migradores são raros, enquanto que espécies não-nativas são comuns ou dominantes.

Palavras-chave: Barragem, Ictiofauna, Longitudinal, Não-nativo, Peixes migradores.

\section{Introduction}

Hydropower dams affect the functioning of main rivers in South America (Agostinho et al., 2016), resulting in a number of negative impacts on biodiversity, especially fishes (Pringle et al., 2000; Agostinho et al., 2007a). Dams block migratory routes, preventing the completion of life cycles (Godinho, Kynard, 2008; Pelicice, Agostinho, 2008); they also cause profound changes in river hydrology and physical/chemical processes that are key to the functioning of fluvial ecosystems (Poff et al., 1997). As a result, the structure of fish assemblages in impounded areas experience strong shifts. The diversity of

\footnotetext{
${ }^{1}$ Núcleo de Estudos Ambientais, Universidade Federal do Tocantins, 77500-000 Porto Nacional, TO, Brazil. fmpelicice@gmail.com, OChttps://orcid.org/0000-0002-9700-1194 (corresponding author)

${ }^{2}$ Departamento de Zoologia, Universidade Estadual Paulista “Júlio de Mesquita Filho”, 18618-970 Botucatu, SP, Brazil. valter.ecologia@gmail.com

${ }^{3}$ Departamento de Biologia, Universidade de São Paulo, 14040-901 Ribeirão Preto, SP, Brazil. (ALHE) andre.esguicero@gmail.com, (MSA) marcifa@usp.br

${ }^{4}$ Núcleo de Pesquisas em Limnologia, Ictiologia e Aquicultura, Universidade Estadual de Maringá, 87020-900 Maringá, PR, Brazil. agostinhoaa@gmail.com
} 
migratory and rheophilic fishes decline (Petrere Jr., 1996; Hoeinghaus et al., 2009; Petesse, Petrere Jr., 2012; Santos et al., 2017), while opportunistic (lentic-adapted) and nonnative species, benefited by the impoundment, spread and dominate assemblages (Pelicice, Agostinho, 2009; Espínola et al., 2010; Britton, Orsi, 2012; Vitule et al., 2012).

The upper Paraná river basin is a special case. This basin is severely regulated by hundreds of large and small dams, which cover almost half of the impounded area in Brazil (Agostinho et al., 2007a). In addition, the main course of all major tributaries (i.e., Paranaíba, Grande, Tietê, Paranapanema) is regulated by cascades of large dams (Agostinho et al., 2016). For example, eleven hydropower dams regulate the main course of the Paranapanema River, creating a cascade of impoundments along the entire river system. Studies that investigated fish diversity in these reservoirs (e.g., Barrella, Petrere Jr., 2003; Hoffmann et al., 2005; Duke Energy, 2008; Orsi, Britton, 2014; Santos et al., 2017; Garcia et al., 2018) indicated that assemblages are composed primarily of smallsized sedentary species, in addition to several non-native fishes. These studies, however, focused on specific impoundments; no study has investigated the whole cascade system, in order to provide a more complete picture about taxonomic diversity and abundance patterns. It is poorly known, for example, whether assemblage structure is homogenized among reservoirs (e.g., Petesse, Petrere Jr., 2012) or contingent to each impoundment. This information is important to guide management and conservation plans, since the Paranapanema basin is severely disturbed by different human activities (e.g., dams, agriculture, urbanization, aquaculture), demanding specific actions to preserve fish diversity and fishing stocks.

In this sense, this study gathered the available information to provide a comprehensive overview of fish diversity in the cascade of reservoirs along the Paranapanema River.
Our main objective was to reveal the structure of fish assemblages in these impoundments, investigating spatial patterns of species richness, composition and abundance of native (non-migratory and migratory) and non-native fishes. In particular, we predict that non-migratory and non-native fishes dominate impoundments in terms of species richness and abundance, as evidenced by previous studies (e.g., Barrella, Petrere Jr., 2003; Petesse, Petrere Jr., 2012; Agostinho et al., 2016). We also expected a trend of increasing species richness, together with changes in composition, along the cascade - a common pattern in large river systems (e.g., Oberdorff et al., 1993; Araújo et al., 2009). Finally, we predict that migratory species are rare (abundance and richness) in impoundments, especially in segments where dams are close to each other (middle/upper reaches) and critical habitats are limited or lacking.

\section{Material and Methods}

Study area. The Paranapanema River is a main tributary of the upper Paraná river basin. Its sources are located in São Paulo State, and the river runs west $c a .900 \mathrm{~km}$, mostly along the border between São Paulo and Paraná States, to join the Paraná River downstream. Dozens of hydropower dams were built in the basin during the $20^{\text {th }}$ century. Eleven large dams regulate the main channel (Fig. 1), affecting the upper, middle and lower reaches of the basin. The first large impoundment date back to 1932, but most dams were constructed after 1970 (Tab. 1). There are marked variations in the size of reservoirs; the largest are located in upper and lower reaches (Tab. 1). Fish passages (ladders) were installed in five dams, namely Canoas I, Canoas II, Ourinhos, Paranapanema and Piraju (Arcifa, Esguícero, 2012; Britto, Carvalho, 2013).

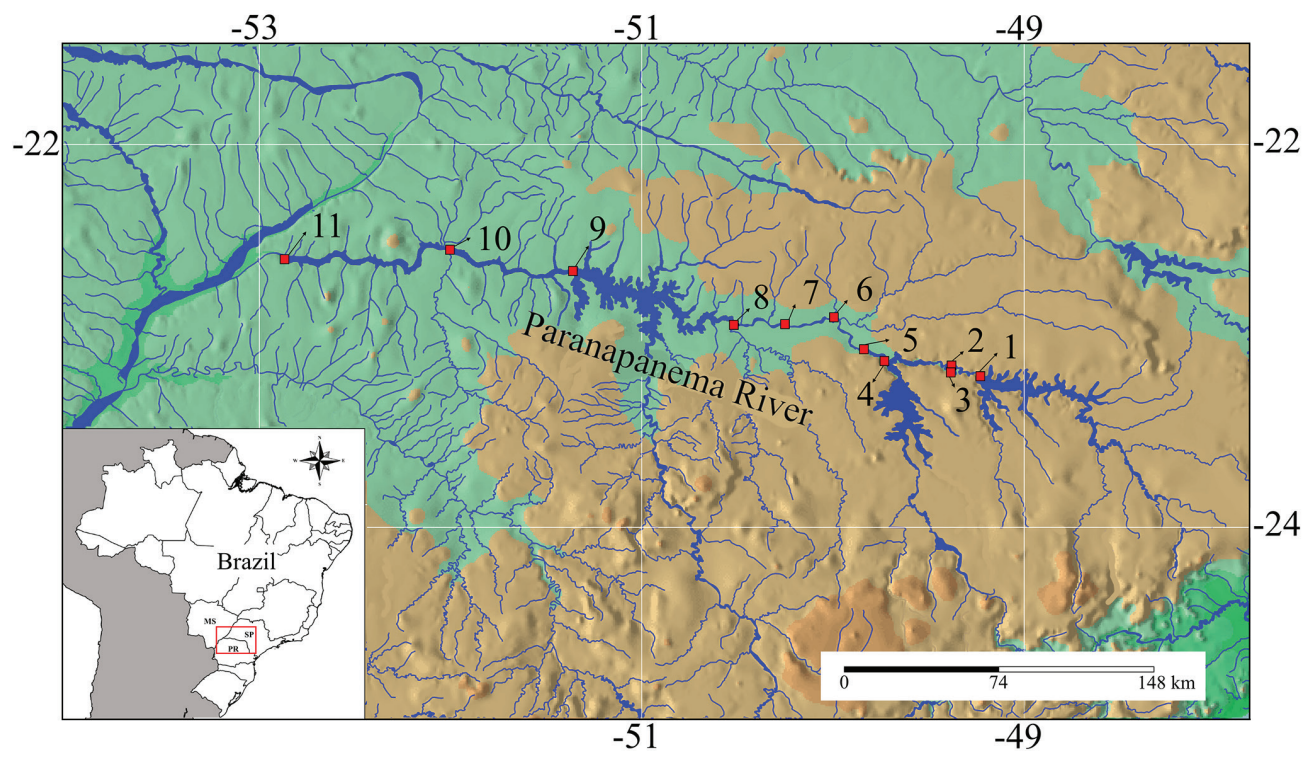

Fig. 1. The cascade of dams (red squares) along the Paranapanema River. Dams: $1=$ Jurumirim; $2=$ Piraju; $3=$ Paranapanema; $4=$ Chavantes; $5=$ Ourinhos; $6=$ Salto Grande; $7=$ Canoas II; $8=$ Canoas I; $9=$ Capivara; $10=$ Taquaruçu; $11=$ Rosana. States: MS = Mato Grosso do Sul; PR = Paraná; $\mathrm{SP}=$ São Paulo. 
Tab. 1. Hydroelectric dams along the Paranapanema River. Information about dams: date of construction, power installed $(\mathrm{MW})$, dam height $(\mathrm{m})$, distance from the mouth $(\mathrm{km})$, presence of fishway. Information about reservoirs: area $\left(\mathrm{km}^{2}\right)$, volume $\left(\mathrm{x} 10^{6} \mathrm{~m}^{3}\right)$, residence time (average days), maximum depth $(\mathrm{m})$ and length $(\mathrm{km})$. Source: Agostinho et al. (2007a); Duke Energy (2008); Pelicice et al. (2015a); websites of hydropower companies. "-" = unavailable information. Basin subdivision in upper, middle and lower reaches followed the Sistema Integrado de Gerenciamento de Recursos Hídricos do Estado de São Paulo (Governo do Estado de São Paulo, 2018).

\begin{tabular}{|c|c|c|c|c|c|c|c|c|c|c|c|c|}
\hline \multirow{2}{*}{ Reservoir } & \multirow{2}{*}{ Sequence } & \multirow{2}{*}{ Reach } & \multicolumn{5}{|c|}{ Dam } & \multicolumn{5}{|c|}{ Reservoir } \\
\hline & & & Date & Power & Height & Distance & Fishway & Area & Volume & Residence & Depth & Length \\
\hline Jurumirim & 1 & Upper & 1962 & 98 & 35 & 527 & & 546 & 7900 & 334 & 40 & 90 \\
\hline Piraju & 2 & Upper & 2002 & 80 & 38 & 496 & ladder & 12.8 & 105 & - & - & 22 \\
\hline Paranapanema & 3 & Upper & 1932 & 31 & 16 & 487 & ladder & 1.5 & - & - & - & 9 \\
\hline Chavantes & 4 & Upper & 1970 & 414 & 89 & 439 & & 400 & 8795 & 353 & 78 & 40 \\
\hline Ourinhos & 5 & Middle & 2005 & 33 & - & 422 & ladder & 5 & 20.8 & 1 & 9 & 6 \\
\hline Salto Grande & 6 & Middle & 1958 & 74 & 25 & 388 & & 12 & 44.2 & 1.4 & 9.2 & 15 \\
\hline Canoas II & 7 & Middle & 1998 & 72 & 25 & 354 & ladder & 22.5 & 140 & 4.4 & 16.5 & 30 \\
\hline Canoas I & 8 & Middle & 1998 & 81 & 29 & 320 & ladder & 30.8 & 207 & 6 & 26 & 30 \\
\hline Capivara & 9 & Middle & 1975 & 619 & 59 & 212 & & 576 & 10540 & 126.8 & 52.5 & 110 \\
\hline Taquaruçu & 10 & Lower & 1992 & 526 & 61 & 134 & & 105.5 & 672.5 & 7.9 & 26.5 & 60 \\
\hline Rosana & 11 & Lower & 1986 & 353 & 30 & 25 & & 220 & 1920 & 18.6 & 26 & 90 \\
\hline
\end{tabular}

Data collection. We gathered data on fish diversity (species richness, composition and abundance) from studies conducted in the basin (Tab. 2). We selected only studies conducted in the impounded area (i.e., lentic and semi-lentic habitats) and adjacent environments directly affected by the impoundment (i.e., upstream stretches, tributaries, lateral lakes, and fish passage facilities). We excluded studies or data collected in environments not affect by the impoundment (i.e., free-flowing streams and rivers), in addition to sites downstream from the dam. We searched for studies in different databases (Web of Science, Scopus, SciELO and Google), private libraries, and performed cross-reference searches. We performed exhaustive searches, and used different keywords in databases (e.g., fish, ichthyofauna, diversity, Paranapanema, Paraná). The survey collected published references (scientific journals and book chapters, $78 \%$ ) and unpublished material (theses, reports and databases, 22\%) (Tab. 2).

In total, we gathered 34 independent studies that investigated fish diversity in nine reservoirs, between 1991 and 2012 (Tab. 2). Some studies investigated more than one reservoir, so our sample size totaled 47 samples. Sampling effort was distributed heterogeneously: Jurumirim, Salto Grande, Capivara, and Rosana received more effort, while Ourinhos and Taquaruçu were less investigated. We found no study conducted in Piraju and Paranapanema reservoirs. Fish sampling employed different gears, but gill nets and seines prevailed (Tab. 2). Most samples (77\%) were collected at least 10 years after the construction of the impoundment. It means that fish assemblages have responded to reservoir conditions and community structure have shifted to another regime and stability (Agostinho et al., 2007a). Our study, therefore, focus on reservoir fish assemblages.

We obtained species lists from each sample, which were organized to build a "sample x species" matrix. Each sample was considered an independent unit (Tab. 2), and we only considered taxa identified to the species level; those reported as "sp." or "spp." were discarded. Species names and taxonomic classification were carefully checked and, when necessary, updated and corrected following Froese, Pauly (2016), Eschmeyer et al. (2017), Eschmeyer, Fong (2017) and recent literature; updates are shown on Tab. S1 (Available only as online supplementary file accessed with the online version of the article at http://www.scielo.br/ni). We then assigned species as native (non-migratory or long distance migratory) and non-native. We used pertinent literature to assign species as long distance migratory, i.e., those that migrate more than $100 \mathrm{~km}$ (sensu Agostinho et al., 2003). Equally, we followed scientific literature to assign species as non-native, i.e., species introduced to the Paranapanema river basin (sensu Reis et al., 2003; Graça, Pavanelli, 2007; Julio Jr. et al., 2009; Britton, Orsi, 2012; Garavello et al., 2012; Orsi, Britton, 2014; Ortega et al., 2015; Froese, Pauly, 2016; Garcia et al., 2018). Abundance data was recorded whenever available (Tab. 2), and used to calculate species relative abundances (\%).

Data analysis. Analyses focused on three groups: (i) native non-migratory species (NT); (ii) native long-distance migratory species (LDM); and (iii) non-native species (NNS). All analyses considered these three groups separately.

To investigate total taxonomic richness in the impoundments, we calculated species accumulation curves based on sampling effort $(n=47)$. Sample order in the original matrix (sample $\mathrm{x}$ species) was randomized 500 times, building expected curves for native (NT + LDM) and non-native species (NNS). We also calculated three nonparametric estimators to estimate total richness: Chao-1, ICE (Incidence-based Coverage Estimator), and Jackknife. These analyses were conducted in the software EstiMateS 5.0 (Colwell, 2006). 
Tab. 2. Samples collected from 34 studies that investigated fish diversity in the cascade of reservoirs along the Paranapanema River. For each sample we provide the sampling period, reservoir age, target environment ( $\mathrm{FL}=$ fish ladder; $\mathrm{LG}=$ lateral lagoon; $\mathrm{RS}=$ reservoir; $\mathrm{RV}=$ river; $\mathrm{TB}=$ tributary $)$, sampling gears $(\mathrm{CN}=$ cast net; $\mathrm{FS}=$ artisanal fishery; $\mathrm{GN}=$ gill nets; $\mathrm{PL}=$ plankton net $\mathrm{OB}=$ observation; $\mathrm{SN}=$ seine; $\mathrm{SV}=$ sieve; $\mathrm{TP}=$ trap $)$, abundance data $(\mathrm{x}=$ available $)$, and data source.

\begin{tabular}{|c|c|c|c|c|c|c|c|}
\hline Samples & Reservoir & Period & Age & Environment & Gear & Abundance & Source \\
\hline 1 & Jurumirim & 1994-1995 & $32-33$ & $\mathrm{RS}$ & GN & $\mathrm{X}$ & Barrella, Petrere Jr. (2003) \\
\hline 2 & Jurumirim & $1995-1996$ & $33-34$ & LG, RS & GN, SV & $\mathrm{X}$ & Carvalho et al. (1998) \\
\hline 3 & Jurumirim & $1996-1997$ & $34-35$ & LG, RS, TB & GN & & Carvalho, Silva (1999) \\
\hline 4 & Jurumirim & 1998-1999 & $36-37$ & LG & SN, SV & $\mathrm{X}$ & Carvalho et al. (2005) \\
\hline 5 & Jurumirim & $2005-2006$ & $43-44$ & LG, RS, TB & FS & & Novaes, Carvalho (2009) \\
\hline 6 & Jurumirim & $2005-2006$ & $43-44$ & LG, RS, TB & FS & & Novaes, Carvalho (2013) \\
\hline 7 & Jurumirim & 1991 & 29 & RS, TB & GN & & Carvalho et al. (1991) apud Kurchevski, Carvalho (2014) \\
\hline 8 & Jurumirim & 2003 & 41 & LG, RS, RV & GN, SN, SV & & Carvalho et al. (2003) apud Kurchevski, Carvalho (2014) \\
\hline 9 & Jurumirim & 2007 & 45 & $\mathrm{RS}$ & GN & & Zanatta (2007) apud Kurchevski, Carvalho (2014) \\
\hline 10 & Jurumirim & 2012 & 50 & LG, RS, RV & GN, SN, SV & $\mathrm{X}$ & Kurchevski (2012) apud Kurchevski, Carvalho (2014) \\
\hline 11 & Chavantes & 2001 & 30 & RS & GN & $\mathrm{X}$ & Unpublished data \\
\hline 12 & Chavantes & $2005-2006$ & $35-36$ & $\mathrm{RS}$ & GN, SN, SV & $\mathrm{X}$ & Magnoni (2009) \\
\hline 13 & Chavantes & $2010-2011$ & $40-41$ & $\mathrm{RS}$ & GN & $\mathrm{X}$ & Caetano et al. (2011) \\
\hline 14 & Ourinhos & 2006-2009 & $1-4$ & LG, RS, TB & $\mathrm{CN}, \mathrm{GN}, \mathrm{SN}, \mathrm{SV}$ & $\mathrm{X}$ & CBA (2009) \\
\hline 15 & Salto Grande & 2001 & 43 & $\mathrm{RS}$ & GN & $\mathrm{X}$ & Unpublished data \\
\hline 16 & Salto Grande & $1992-1993$ & $34-35$ & RS, RV & GN & & Dias, Garavello (1998) \\
\hline 17 & Salto Grande & 1993-1995 & $35-37$ & RS, RV & GN & & $\operatorname{Dias}(2003)$ \\
\hline 18 & Salto Grande & $2005-2006$ & $47-48$ & LG, RS & GN, SN, SV & $\mathrm{X}$ & Brandão et al. (2009) \\
\hline 19 & Salto Grande & $2005-2006$ & $47-48$ & LG & GN & & Vianna (2008) \\
\hline 20 & Canoas II & 2001 & 3 & RS & GN & $\mathrm{X}$ & Unpublished data \\
\hline 21 & Canoas II & $2000-2001$ & $2-3$ & RS, LG, FL & $\mathrm{SN}$ & & Britto, Sirol (2007) \\
\hline 22 & Canoas II & $2000-2006$ & $2-8$ & RS, LG, FL & SN, SV & $\mathrm{X}$ & Britto (2009) \\
\hline 23 & Canoas II & 2001-2002 & $2-3$ & RS, LG, FL & $\mathrm{SN}$ & $\mathrm{X}$ & Britto, Carvalho (2013) \\
\hline 24 & Canoas I & 2001 & 3 & RS & GN & $\mathrm{X}$ & Unpublished data \\
\hline 25 & Canoas I & $2000-2001$ & $2-3$ & RS, LG, FL & $\mathrm{SN}$ & & Britto, Sirol (2007) \\
\hline 26 & Canoas I & $2000-2006$ & $2-8$ & RS, LG, FL & SN, SV & $\mathrm{X}$ & Britto (2009) \\
\hline 27 & Canoas I & 2001-2002 & $2-3$ & RS, LG, FL & $\mathrm{SN}$ & $\mathrm{X}$ & Britto, Carvalho (2013) \\
\hline 28 & Capivara & 2001 & 26 & RS & GN & $\mathrm{X}$ & Unpublished data \\
\hline 29 & Capivara & $1992-1993$ & $17-18$ & TB & GN & $\mathrm{X}$ & Bennemann et al. (2000) \\
\hline 30 & Capivara & $2001-2002$ & $26-27$ & TB, RS & GN & $\mathrm{X}$ & Bennemann et al. (2013) \\
\hline 31 & Capivara & 1993-1995 & $17-19$ & RS & GN & & Dias (2003) \\
\hline 32 & Capivara & 1994-1995 & $18-19$ & $\mathrm{RS}$ & GN & $\mathrm{X}$ & Barrella, Petrere Jr. (2003) \\
\hline 33 & Capivara & 2001-2002 & $26-27$ & RS & $\mathrm{CN}, \mathrm{GN}, \mathrm{SN}, \mathrm{SV}$ & $\mathrm{X}$ & Hoffmann et al. (2005) \\
\hline 34 & Capivara & 2001-2002 & $26-27$ & TB & $\mathrm{CN}, \mathrm{GN}$ & $\mathrm{X}$ & Shibatta et al. (2007) \\
\hline 35 & Capivara & $2003-2004$ & $28-29$ & TB & GN & & Vianna, Nogueira (2008) \\
\hline 36 & Capivara & 1990-1994 & $15-19$ & RS & GN & & Orsi, Britton (2014) \\
\hline 37 & Capivara & $2001-2010$ & $26-35$ & $\mathrm{RS}$ & GN & & Orsi, Britton (2014) \\
\hline 38 & Taquaruçu & 2001 & 9 & $\mathrm{RS}$ & GN & $\mathrm{X}$ & Unpublished data \\
\hline 39 & Taquaruçu & $1993-2000$ & $1-8$ & $\mathrm{RS}$ & GN & $\mathrm{X}$ & Britto, Carvalho (2006) \\
\hline 40 & Rosana & 2001 & 15 & RS & GN & $\mathrm{X}$ & Unpublished data \\
\hline 41 & Rosana & 1994-1995 & $8-9$ & $\mathrm{RS}$ & GN & $\mathrm{X}$ & Barrella, Petrere Jr. (2003) \\
\hline 42 & Rosana & 1997-1998 & $11-12$ & TB & GN & $\mathrm{X}$ & Abilhôa, Bastos (2005) \\
\hline 43 & Rosana & $2000-2001$ & $14-15$ & RS & $\mathrm{SN}, \mathrm{OB}$ & $\mathrm{X}$ & Casatti et al. (2003) \\
\hline 44 & Rosana & 2002 & 16 & RS, RV, TB & $\mathrm{SN}, \mathrm{PL}$ & $\mathrm{X}$ & Kipper et al. (2011) \\
\hline 45 & Rosana & 2003 & 17 & RS, TB & $\mathrm{TP}$ & $\mathrm{X}$ & Pelicice et al. (2005) \\
\hline 46 & Rosana & $2004-2005$ & $18-19$ & LG & SN & $\mathrm{X}$ & Ferrareze, Nogueira (2011) \\
\hline 47 & Rosana & $2005-2007$ & $19-21$ & RS & SN, TP & $\mathrm{X}$ & Pelicice, Agostinho (2009); Pelicice et al. (2015b) \\
\hline
\end{tabular}


To investigate spatial variation in species richness and composition, we pooled the 47 samples within each reservoir to build a "reservoir x species" matrix. We then calculated total richness per reservoir for each group (NT, LDM and NNS). To investigate spatial variations in species composition, we used Non-Metric Multidimensional Scaling (NMDS) based on Jaccard distance (presence/absence). We conducted three NMDS, one for each group. These analyses were carried out in the software Past 1.75b (Hammer et al., 2001).

We also investigated trends in abundance. Twenty-two studies (Tab. 2) provided abundance data for all species in the assemblage (number, density or capture per effort); we used these data to calculate the relative abundance (\%) of each species, generating an abundance-based "sample $\mathrm{x}$ species" matrix (32 samples). We used this matrix to investigate spatial variation in the relative abundance of LDM and NNS in assemblages, by plotting samples against reservoirs (scatterplot). We also calculated the range of relative abundance (minimum-maximum) for each species along the cascade system.

\section{Results}

We recorded 161 fish species in the cascade of reservoirs along the Paranapanema River, being 111 native and 50 non-native to the basin (Tab. 3). Most species belonged to the order Characiformes, followed by Siluriformes and Cichliformes.

Tab. 3. Fish species recorded in the cascade of reservoirs along the Paranapanema River. LDM = long distance migratory; NNS = non-native. Taxonomic classification followed Eschmeyer, Fong (2017).

\begin{tabular}{|c|c|c|}
\hline Species & LDM & NNS \\
\hline \multicolumn{3}{|l|}{ MYLIOBATIFORMES } \\
\hline \multicolumn{3}{|l|}{ Potamotrygonidae } \\
\hline Potamotrygon cf. motoro (Müller \& Henle, 1841) & & $\mathrm{X}$ \\
\hline \multicolumn{3}{|l|}{ CYPRINIFORMES } \\
\hline \multicolumn{3}{|l|}{ Cyprinidae } \\
\hline Cyprinus carpio Linnaeus, 1758 & & $\mathrm{X}$ \\
\hline \multicolumn{3}{|l|}{ CHARACIFORMES } \\
\hline \multicolumn{3}{|l|}{ Crenuchidae } \\
\hline \multicolumn{3}{|l|}{ Characidium fasciatum Reinhardt, 1867} \\
\hline \multicolumn{3}{|l|}{ Characidium zebra Eigenmann, 1909} \\
\hline \multicolumn{3}{|l|}{ Erythrinidae } \\
\hline Hoplias intermedius (Günther, 1864) & & $X$ \\
\hline Hoplias lacerdae Miranda Ribeiro, 1908 & & $\mathrm{X}$ \\
\hline \multicolumn{3}{|l|}{ Hoplias malabaricus (Bloch, 1794) } \\
\hline \multicolumn{3}{|l|}{ Parodontidae } \\
\hline \multicolumn{3}{|l|}{ Apareiodon affinis (Steindachner, 1879) } \\
\hline \multicolumn{3}{|l|}{ Apareiodon ibitiensis Amaral Campos, 1944} \\
\hline \multicolumn{3}{|l|}{ Apareiodon piracicabae (Eigenmann, 1907) } \\
\hline \multicolumn{3}{|l|}{ Parodon nasus Kner, 1859} \\
\hline \multicolumn{3}{|l|}{ Cynodontidae } \\
\hline Rhaphiodon vulpinus Spix \& Agassiz, 1829 & $\mathrm{X}$ & \\
\hline
\end{tabular}

Tab. 3. (continued).

\begin{tabular}{|c|c|c|}
\hline Species & LDM & NNS \\
\hline \multicolumn{3}{|l|}{ Serrasalmidae } \\
\hline Metynnis lippincottianus Cope, 1870 & & $\mathrm{X}$ \\
\hline \multicolumn{3}{|l|}{ Metynnis mola Eigenmann \& Kennedy, 1903} \\
\hline \multicolumn{3}{|l|}{ Myloplus levis (Eigenmann \& McAtee, 1907) } \\
\hline \multicolumn{3}{|l|}{ Myloplus tiete (Eigenmann \& Norris, 1900) } \\
\hline Piaractus mesopotamicus (Holmberg, 1887) & $\mathrm{X}$ & \\
\hline \multicolumn{3}{|l|}{ Serrasalmus maculatus Kner, 1858} \\
\hline Serrasalmus marginatus Valenciennes, 1837 & & $\mathrm{X}$ \\
\hline \multicolumn{3}{|l|}{ Anostomidae } \\
\hline \multicolumn{3}{|l|}{ Leporellus pictus (Kner, 1858) } \\
\hline \multicolumn{3}{|l|}{ Leporellus vittatus (Valenciennes, 1850) } \\
\hline \multicolumn{3}{|l|}{ Leporinus amblyrhynchus Garavello \& Britski, 1987} \\
\hline \multicolumn{3}{|l|}{ Leporinus friderici (Bloch, 1794) } \\
\hline \multicolumn{3}{|l|}{ Leporinus lacustris Amaral Campos, 1945} \\
\hline \multicolumn{3}{|l|}{ Leporinus octofasciatus Steindachner, 1915} \\
\hline \multicolumn{3}{|l|}{ Leporinus paranensis Garavello \& Britski, 1987} \\
\hline \multicolumn{3}{|l|}{ Leporinus striatus Kner, 1858} \\
\hline Megaleporinus elongatus (Valenciennes, 1850) & $\mathrm{X}$ & \\
\hline Megaleporinus macrocephalus (Garavello \& Britski, 1988) & & $\mathrm{X}$ \\
\hline Megaleporinus obtusidens (Valenciennes, 1837) & $\mathrm{X}$ & \\
\hline \multicolumn{3}{|l|}{ Schizodon altoparanae Garavello \& Britski, 1990} \\
\hline Schizodon borellii (Boulenger, 1900) & & $\mathrm{X}$ \\
\hline \multicolumn{3}{|l|}{ Schizodon intermedius Garavello \& Britski, 1990} \\
\hline \multicolumn{3}{|l|}{ Schizodon nasutus Kner, 1858} \\
\hline \multicolumn{3}{|l|}{ Curimatidae } \\
\hline \multicolumn{3}{|l|}{ Cyphocharax modestus (Fernández-Yépez, 1948) } \\
\hline \multicolumn{3}{|l|}{ Cyphocharax nagelii (Steindachner, 1881) } \\
\hline Steindachnerina brevipinna (Eigenmann \& Eigenmann, 1889) & & $\mathrm{X}$ \\
\hline Steindachnerina insculpta (Fernández-Yépez, 1948) & & \\
\hline Prochilodontidae & & \\
\hline Prochilodus lineatus (Valenciennes, 1837) & $\mathrm{X}$ & \\
\hline Lebiasinidae & & \\
\hline Pyrrhulina australis Eigenmann \& Kennedy, 1903 & & \\
\hline Triportheidae & & \\
\hline Triportheus angulatus (Spix \& Agassiz, 1829) & & $\mathrm{X}$ \\
\hline Triportheus nematurus (Kner, 1858) & & $\mathrm{X}$ \\
\hline Bryconidae & & \\
\hline Brycon nattereri Günther, 1864 & & \\
\hline Brycon orbignyanus (Valenciennes, 1850) & $\mathrm{X}$ & \\
\hline Salminus brasiliensis (Cuvier, 1816) & $\mathrm{X}$ & \\
\hline Salminus hilarii Valenciennes, 1850 & $\mathrm{X}$ & \\
\hline Acestrorhynchidae & & \\
\hline Acestrorhynchus lacustris (Lütken, 1875) & & \\
\hline Characidae & & \\
\hline Aphyocharax anisitsi Eigenmann \& Kennedy, 1903 & & \\
\hline Aphyocharax dentatus Eigenmann \& Kennedy, 1903 & & $\mathrm{X}$ \\
\hline Astyanax bockmanni Vari \& Castro, 2007 & & \\
\hline Astyanax fasciatus (Cuvier, 1819) & & \\
\hline Astyanax lacustris (Lütken, 1875) & & \\
\hline Astyanax paranae Eigenmann, 1914 & & \\
\hline Bryconamericus iheringii (Boulenger, 1887) & & \\
\hline ‘Cheirodon’stenodon Eigenmann, 1915 & & \\
\hline Piabarchus stramineus (Eigenmann, 1908) & & \\
\hline Galeocharax gulo (Cope, 1870) & & \\
\hline Hemigrammus marginatus Ellis, 1911 & & \\
\hline
\end{tabular}


Tab. 3. (continued).

\begin{tabular}{l}
\hline Species \\
\hline Hyphessobrycon anisitsi (Eigenmann, 1907) \\
Hyphessobrycon bifasciatus Ellis, 1911 \\
Hyphessobrycon eques (Steindachner, 1882) \\
Moenkhausia intermedia Eigenmann, 1908 \\
Odontostilbe weitzmani Chuctaya, Bührnheim \& Malabarba, 2018 \\
Oligosarcus paranensis Menezes \& Géry, 1983 \\
Oligosarcus pintoi Amaral Campos, 1945 \\
Piabina argentea Reinhardt, 1867 \\
Roeboides descalvadensis Fowler, 1932 \\
Planaltina britskii Menezes, Weitzman \& Burns, 2003 \\
Serrapinnus heterodon (Eigenmann, 1915) \\
Serrapinnus notomelas (Eigenmann, 1915) \\
GYMNOTIFORMES \\
Gymnotidae \\
Gymnotus cf. carapo Linnaeus, 1758 \\
Gymnotus inaequilabiatus (Valenciennes, 1839) \\
Gymnotus sylvius Albert \& Fernandes-Matioli, 1999 \\
Rhamphichthyidae
\end{tabular}

Rhamphichthys hahni (Meinken, 1937)

Hypopomidae

Brachyhypopomus gauderio Giora \& Malabarba, 2009

Sternopygidae

Eigenmannia cf. trilineata López \& Castello, 1966

Eigenmannia virescens (Valenciennes, 1836)

Sternopygus macrurus (Bloch \& Schneider, 1801)

Apteronotidae

Apteronotus cf. caudimaculosus de Santana, 2003

Apteronotus brasiliensis (Reinhardt, 1852)

Apteronotus ellisi (Alonso de Arámburu, 1957)

\section{SILURIFORMES}

\section{Cetopsidae}

Cetopsis gobioides Kner, 1858

\section{Auchenipteridae}

Ageneiosus militaris Valenciennes, 1836

Auchenipterus osteomystax (Miranda Ribeiro, 1918)

Tatia intermedia (Steindachner, 1877)

Tatia neivai (Ihering, 1930)

Trachelyopterus galeatus (Linnaeus, 1766)

\section{Doradidae}

Ossancora eigenmanni (Boulenger, 1895)

Pterodoras granulosus (Valenciennes, 1821)

Rhinodoras dorbignyi (Kner, 1855)

Trachydoras paraguayensis (Eigenmann \& Ward, 1907)

\section{Heptapteridae}

Imparfinis schubarti (Gomes, 1956)

Phenacorhamdia tenebrosa (Schubart, 1964)

Pimelodella avanhandavae Eigenmann, 1917

Pimelodella gracilis (Valenciennes, 1835)

Pimelodella meeki Eigenmann, 1910

Rhamdia cf. quelen (Quoy \& Gaimard, 1824)

\section{Pimelodidae}

Hypophthalmus edentatus Spix \& Agassiz, 1829

Iheringichthys labrosus (Lütken, 1874)

Megalonema platanum (Günther, 1880)
Tab. 3. (continued).

LDM NNS

Species

LDM NNS

Pimelodus absconditus Azpelicueta, 1995

Pimelodus maculatus Lacepède, 1803

$\mathrm{X}$

X Pimelodus microstoma Steindachner, 1877

Pimelodus ornatus Kner, 1858

$\mathrm{X}$

X Pimelodus paranaensis Britski \& Langeani, 1988

Pinirampus pirinampu (Spix \& Agassiz, 1829)

Pseudoplatystoma corruscans (Spix \& Agassiz, 1829)

Pseudoplatystoma fasciatum (Linnaeus, 1766)

$\mathrm{X}$

Sorubim lima (Bloch \& Schneider, 1801)

Steindachneridion scriptum (Miranda-Ribeiro, 1918)

Zungaro jahu (Ihering, 1898)

Pseudopimelodidae

Pseudopimelodus mangurus (Valenciennes, 1835)

\section{Clariidae}

Clarias gariepinus (Burchell, 1822)

\section{Trichomycteridae}

Trichomycterus diabolus Bockmann, Casatti \& de Pinna, 2004

\section{Callichthyidae}

$\mathrm{X}$

Callichthys callichthys (Linnaeus, 1758)

Corydoras aeneus (Gill, 1858)

X

Corydoras ehrhardti Steindachner, 1910

Corydoras paleatus (Jenyns, 1842)

$\mathrm{X}$

Hoplosternum littorale (Hancock, 1828)

\section{Loricariidae}

Hypostomus albopunctatus (Regan, 1908)

Hypostomus ancistroides (Ihering, 1911)

X Hypostomus auroguttatus Kner, 1854

Hypostomus hermanni (Ihering, 1905)

Hypostomus iheringi (Regan, 1908)

Hypostomus margaritifer (Regan, 1908)

Hypostomus multidens Jerep, Shibatta, Zawadzki, 2007

Hypostomus myersi (Gosline, 1947)

Hypostomus nigromaculatus (Schubart, 1964)

Hypostomus paulinus (Ihering, 1905)

X Hypostomus regani (Ihering, 1905)

X Hypostomus roseopunctatus Reis, Weber \& Malabarba, 1990

Hypostomus strigaticeps (Regan, 1908)

X

Hypostomus ternetzi (Boulenger, 1895)

$\mathrm{X}$

Hypostomus tietensis (Ihering, 1905)

X Hypostomus topavae (Godoy, 1969)

X X Hypostomus variostictus (Miranda Ribeiro, 1912)

X

Loricaria simillima Regan, 1904

X Loricariichthys labialis (Boulenger, 1895)

Loricariichthys platymetopon Isbrücker \& Nijssen, 1979

Megalancistrus parananus (Peters, 1881)

Paraloricaria vetula (Valenciennes, 1835)

X Proloricaria prolixa (Isbrücker \& Nijssen, 1978)

Pterygoplichthys ambrosettii (Holmberg, 1893)

Rhinelepis aspera Spix \& Agassiz 1829

SYNBRANCHIFORMES

\section{Synbranchidae}

X

Synbranchus cf. marmoratus Bloch, 1795

PLEURONECTIFORMES

\section{Achiridae}


Tab. 3. (continued).

\begin{tabular}{|c|c|c|}
\hline Species & LDM & NNS \\
\hline Catathyridium jenynsii (Günther, 1862) & & $\mathrm{X}$ \\
\hline \multicolumn{3}{|l|}{ CICHLIFORMES } \\
\hline \multicolumn{3}{|l|}{ Cichlidae } \\
\hline Astronotus crassipinnis Heckel, 1840 & & $\mathrm{X}$ \\
\hline Cichla kelberi Kullander \& Ferreira, 2006 & & $\mathrm{X}$ \\
\hline Cichla piquiti Kullander \& Ferreira, 2006 & & $\mathrm{X}$ \\
\hline \multicolumn{3}{|l|}{ Cichlasoma paranaense Kullander, 1983} \\
\hline Coptodon rendalli (Boulenger, 1897) & & $\mathrm{X}$ \\
\hline \multicolumn{3}{|l|}{ Crenicichla britskii Kullander, 1982} \\
\hline \multicolumn{3}{|l|}{ Crenicichla haroldoi Luengo \& Britski, 1974} \\
\hline \multicolumn{3}{|l|}{ Crenicichla jaguarensis Haseman, 1911} \\
\hline \multicolumn{3}{|l|}{ Crenicichla cf. niederleinii (Holmberg, 1891) } \\
\hline \multicolumn{3}{|l|}{ Geophagus brasiliensis (Quoy \& Gaimard, 1824) } \\
\hline Oreochromis niloticus (Linnaeus, 1758) & & $\mathrm{X}$ \\
\hline Satanoperca aff. pappaterra (Heckel, 1840) & & $\mathrm{X}$ \\
\hline \multicolumn{3}{|l|}{ CYPRINODONTIFORMES } \\
\hline \multicolumn{3}{|l|}{ Poeciliidae } \\
\hline \multicolumn{3}{|l|}{ Phalloceros harpagos Lucinda, 2008} \\
\hline Poecilia reticulata Peters, 1859 & & $\mathrm{X}$ \\
\hline \multicolumn{3}{|l|}{ PERCIFORMES } \\
\hline \multicolumn{3}{|l|}{ Sciaenidae } \\
\hline Plagioscion squamosissimus (Heckel, 1840) & & $\mathrm{X}$ \\
\hline
\end{tabular}

Considering only native species $(\mathrm{NT}+\mathrm{LDM}=111$ species), the accumulation curve tended to stabilize (Fig. 2), but non-parametric estimators indicated that total richness is underestimated (Chao- $1=132.4 \pm 13.2$ $\mathrm{SD}$; $\mathrm{ICE}=125.1 \pm 0.01$; Jackknife-1 $=129.6 \pm 7.6$ ). Among native species, we recorded 14 long-distance migratory species (Tab. 3). Considering non-native species $(\mathrm{NNS}=50)$, the accumulation curve also tended to stabilize (Fig. 2), and non-parametric estimators confirmed this trend (Chao- $1=60.1 \pm 7.2 ; \mathrm{ICE}=60.5$ \pm 0.01 ; Jackknife- $1=63.7 \pm 3.7)$. We recorded three nonnative migratory species (Tab. 3 ).

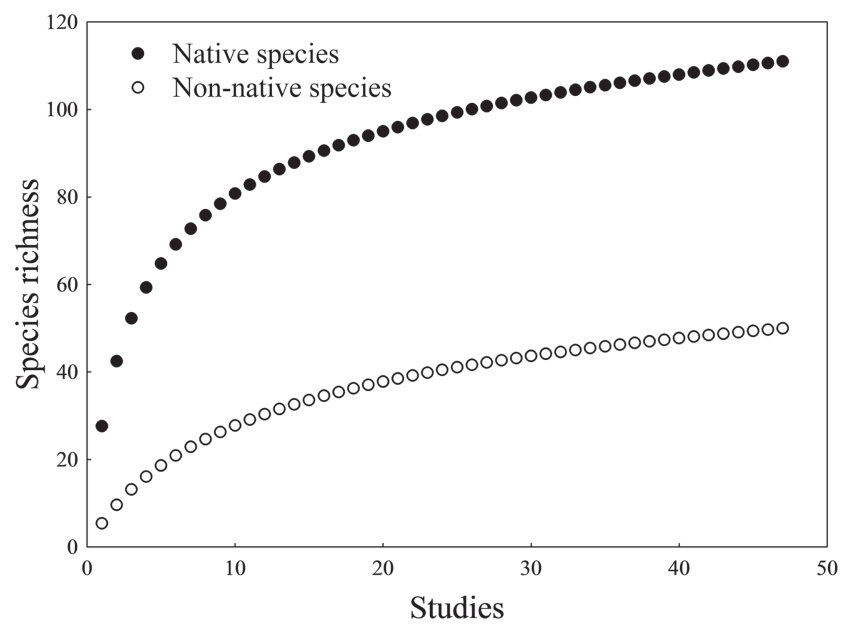

Fig. 2. Species accumulation curves controlled by sampling effort (47 samples). Curves were calculated separately for native and non-native species.
Reservoirs showed a mean species richness of 72 species (range: 56 to 112). Considering only NT, each reservoir had 49.9 species on average (range: 39 to 80 ), with no longitudinal trend along the cascade system (Fig. 3). Migratory species were recorded in all reservoirs, with a mean value of 8.1 species/ reservoir (range: 3 to 12). However, LDM richness showed a clear longitudinal pattern, with higher values in reservoirs located below Salto Grande dam (Fig. 3). Non-native species were also recorded in all reservoirs, with a mean of $14 \mathrm{NNS}$ per reservoir (range: 8 to 23). Reservoirs located in the lower reach, together with Salto Grande, contained more NNS (Fig. 3).

(a)

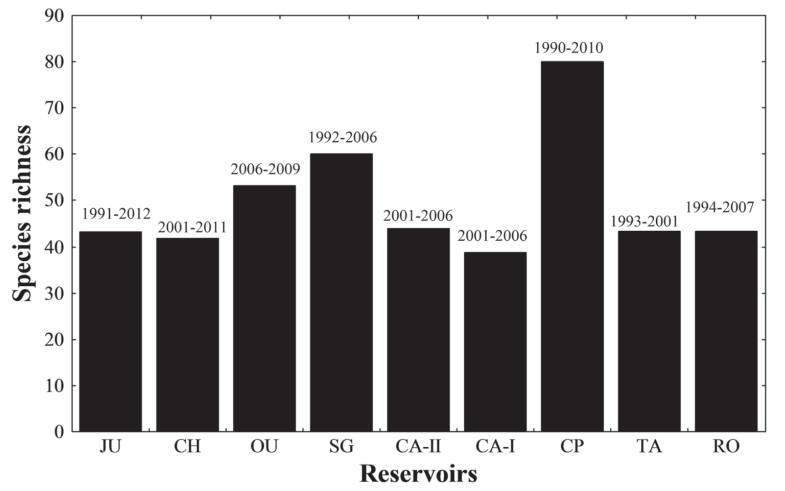

(b)

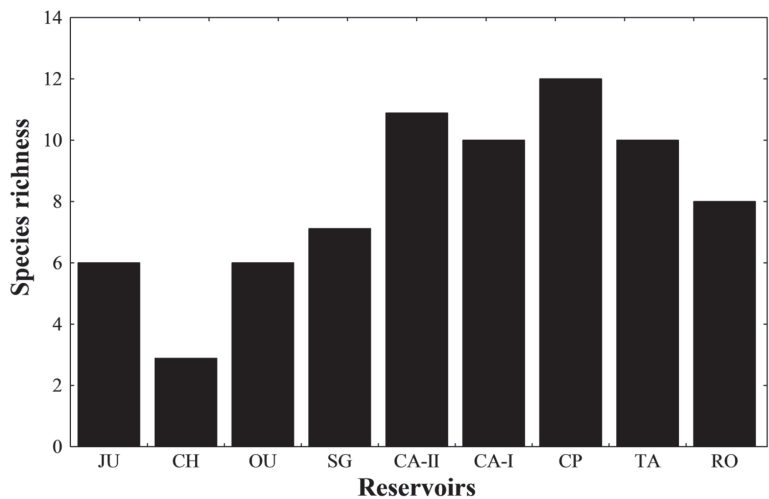

(c)

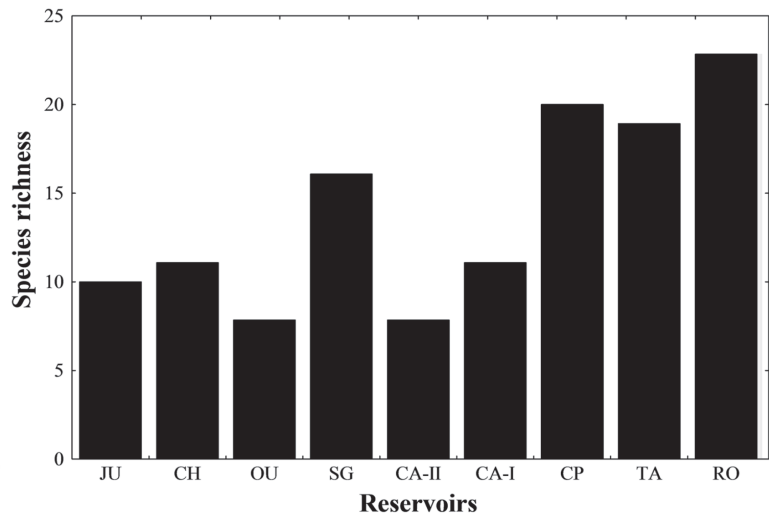

Fig. 3. Species richness in the cascade of reservoirs along the Paranapanema River, separated as native non-migratory (a), native long-distance migratory (b) and non-native species (c). The sampling period is shown above each bar. Reservoir codes: $\mathrm{JU}=$ Jurumirim; $\mathrm{CH}=$ Chavantes; $\mathrm{OU}=$ Ourinhos; $\mathrm{SG}=$ Salto Grande; $\mathrm{CA}-\mathrm{II}=$ Canoas II; CA-I = Canoas I; CP $=$ Capivara; $\mathrm{TA}=$ Taquaruçu; $\mathrm{RO}=$ Rosana . 
Non-Metric Multidimensional Scaling (NMDS; stress = 0.14 ) showed a longitudinal gradient of species composition for NT (Fig. 4a). Jurumirim (first reservoir in the cascade) and Rosana (last in the cascade) were placed in opposing ends along axis 1 (Fig. 4a); some reservoirs located in middle and lower reaches overlapped, indicating similar composition. Many species seemed to be widely distributed in the cascade system, while some were restricted to specific impoundments (Tab. 4). Considering LDM, the NMDS (stress $=0.13$ ) also revealed a longitudinal gradient, but formed two groups: upstream and downstream Salto Grande reservoir (Fig. 4b). The first group was composed of species with wide distribution in the cascade system, while the second group included large-sized species (Tab. 5). Regarding NNS, most reservoirs overlapped in the ordination; because the stress value was higher $(=0.22)$, the plot was not interpreted. Based on Tab. 6, some NNS were widely distributed in the cascade, but no species was present in all reservoirs. Other NNS were restricted to some reservoir or segment, creating a turnover pattern.
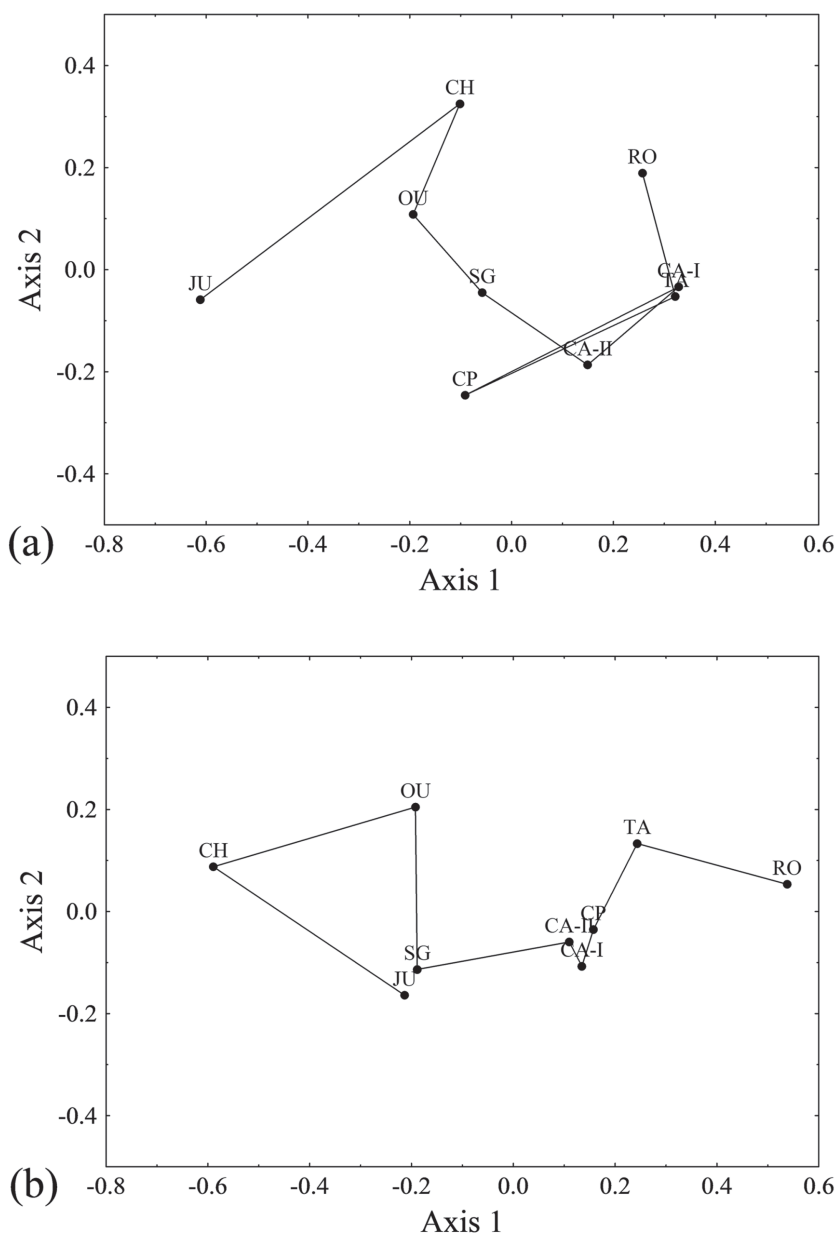

Fig. 4. Non-Metric Multidimensional Scaling (NMDS) showing variation in assemblage composition in the cascade of reservoirs along the Paranapanema River, considering native nonmigratory species (a) and native long-distance migratory species (b). The line connecting reservoirs indicates the sequence in the cascade system. Reservoir names (codes) are shown in Fig. 3.
The most abundant species in the cascade system included small native characids (Tab. 4), such as Astyanax lacustris, Schizodon nasutus, Serrasalmus maculatus, Steindachnerina insculpta and Apareiodon affinis, and the catfish Iheringichthys labrosus. Migratory fishes, on the other hand, showed very low abundance in assemblages (Tab. 5), where Pimelodus maculatus was the most abundant and frequent. In almost all samples (88\%), LDM summed less than $10 \%$ of total abundance (range: 0 to $35.8 \%$ ), with higher values in Canoas I, Canoas II and Capivara reservoirs (Fig. 5a). Differently, NNS were more abundant and summed more than $10 \%$ of total abundance in most samples (range: 0 to $90 \%$ ) (Fig. 5b). Higher values were recorded in reservoirs located downstream (Capivara, Taquaruçu and Rosana). The most abundant species in the cascade system were Plagioscion squamosissimus and Hyphessobrycon eques, in addition to others abundant in specific reservoirs or stretches, such as Roeboides descalvadensis, Serrasalmus marginatus, Loricariichthys platymetopon and Hypophthalmus edentatus (Tab. 6).
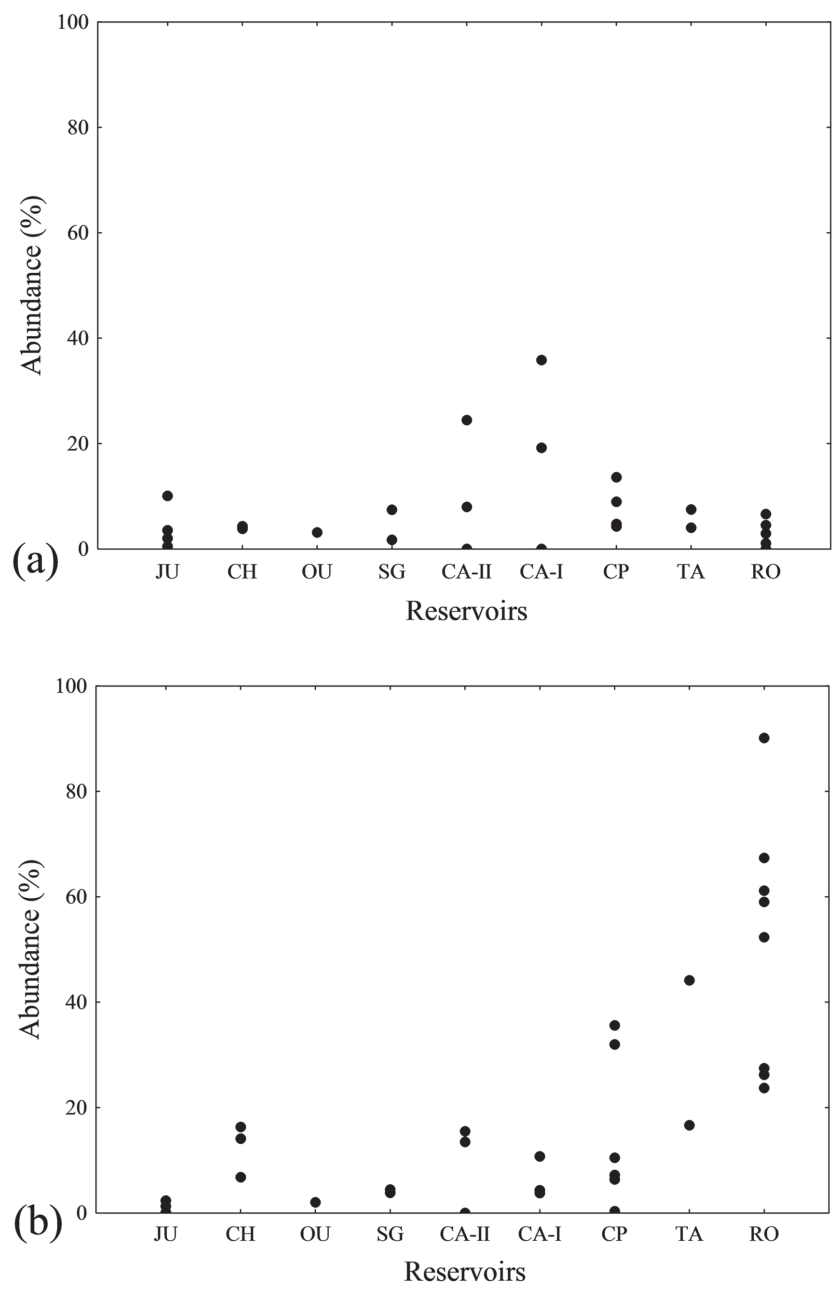

Fig. 5. Relative abundance (\%) of long-distance migratory fishes (a) and non-native species (b) in the cascade of reservoirs along the Paranapanema River. Each dot is a sample $(n=32)$. Reservoir names (codes) are shown in Fig. 3. 
Tab. 4. Distribution of non-migratory native species in the cascade of reservoirs along the Paranapanema River (gray $=$ presence), based on all studies (47 samples). Numbers are the relative abundance range (percentage, min-max), based on studies that provided abundance data (32 samples); the most abundant species in the cascade are highlighted in bold. Reservoir names (codes) are shown in Fig. 3.

\begin{tabular}{|c|c|c|c|c|c|c|c|c|c|}
\hline Species & JU & $\mathrm{CH}$ & OU & SG & CA-II & CA-I & $\mathrm{CP}$ & TA & RO \\
\hline Leporinus amblyrhynchus & $0-0.1$ & $0-0.5$ & 4.3 & $0-0.1$ & 0 & $0-0.4$ & $0-4.3$ & $0-0.1$ & $0-0.3$ \\
\hline Leporinus friderici & $0-1.3$ & $0-0.8$ & 0 & $\begin{array}{ll}1.1 & 0.2\end{array}$ & $0.3-2.5$ & $0.2-5.1$ & $0.2-1.8$ & $0.2-1.7$ & $0-1.2$ \\
\hline Leporinus octofasciatus & $0-0.2$ & $0-0.3$ & 0.2 & $0-0.1$ & $0-1.3$ & $0-2.3$ & $0-1.1$ & $0-0.6$ & $0-0.1$ \\
\hline Schizodon nasutus & $0.1-7.7$ & $1.9-7.6$ & 2.4 & $4.2-9.8$ & $1.5-3.9$ & $0.3-6.1$ & $1.2-2.8$ & $3.2-7.6$ & 0 - 2.0 \\
\hline Astyanax lacustris & 0 - 11.5 & $7.4-34.8$ & 4.9 & $9.4-24.9$ & $4.2-8.0$ & $2.3-7.0$ & $1.4-32.2$ & $1.0-3.2$ & 0 - 3.1 \\
\hline Galeocharax gulo & $0-1.8$ & $1.4-7.6$ & 2.0 & $1.3-1.7$ & $0-3.1$ & $0-1.9$ & $0-0.7$ & $0-0.3$ & $0-0.1$ \\
\hline Serrasalmus maculatus & $2.0-10.6$ & $1.1-3.6$ & 3.7 & $4.5-5.2$ & $0.9-3.7$ & $0.6-1.2$ & 0 - 3.6 & $0.2-1.5$ & 0 - 11.1 \\
\hline Cyphocharax modestus & $7.4-76.9$ & $0-0.2$ & 1.6 & $0-1.7$ & $0-0.9$ & $0-0.1$ & $0-0.5$ & $0-1.2$ & $0-2.1$ \\
\hline Steindachnerina insculpta & $1.9-9.4$ & 0 - 13.6 & 17.1 & 0 - 19.2 & $0-0.4$ & $0-0.2$ & 0 - 8.1 & $0-8.3$ & $0-7.7$ \\
\hline Hoplias malabaricus & $0.5-2.8$ & $0-1.1$ & 1.2 & $0.3-1.6$ & $0-0.9$ & $0-0.8$ & $0.1-1.4$ & $0.2-1.3$ & $0-8.4$ \\
\hline Apareiodon affinis & $0-5.0$ & $0-52.5$ & 19.8 & $2.2-3.8$ & $0-37.0$ & 0 - 11.1 & 0 - 30.2 & $1.3-8.0$ & 0 - 8.1 \\
\hline Geophagus brasiliensis & $0-0.6$ & $0.4-1.7$ & 1.8 & $0.5-4.1$ & $0-0.2$ & $0-0.6$ & $0-1.2$ & $0-0.2$ & $0-3.8$ \\
\hline Hypostomus ancistroides & $0-2.6$ & $0-0.1$ & 1.2 & $0-0.5$ & $0-0.4$ & $0-0.6$ & $0-1.8$ & $0.7-0.8$ & $0-0.6$ \\
\hline Iheringichthys labrosus & $0-0.6$ & $1.1-2.8$ & 1.9 & $6.6-35.7$ & 0 - 14.5 & 0 - 24.4 & $1.6-5.9$ & $5.9-9.2$ & $0-4.1$ \\
\hline Astyanax fasciatus & $0.5-5.1$ & $0-8.1$ & 2.6 & $0.3-4.3$ & $0-1.8$ & $0-0.4$ & $0-7.9$ & $0-0.1$ & \\
\hline Serrapinnus notomelas & 0 - 52.6 & $0-0.3$ & $\mathbf{0}$ & $\mathbf{0}$ & $0-21.3$ & 0 - 12.7 & $0-0.9$ & & $0-9.2$ \\
\hline Hypostomus strigaticeps & $0-0.1$ & $0-0.1$ & 5.5 & $0-0.7$ & $0-0.2$ & & $0-0.1$ & & \\
\hline Gymnotus cf. carapo & $0-0.2$ & $0-0.7$ & 0 & $0-0.4$ & & $0-0.4$ & $0-0.4$ & $0-0.2$ & $0-0.6$ \\
\hline Eigenmannia $\mathrm{cf}$. trilineata & $0-0.1$ & $0-0.4$ & 0 & $0-1.7$ & & & $0-0.1$ & & $0-2.7$ \\
\hline Rhamdia cf. quelen & $0-0.5$ & $0-0.1$ & 0.2 & $0.3-0.5$ & & & $0-5.8$ & & $0-0.3$ \\
\hline Piabina argentea & $0-0.1$ & $0-0.3$ & 0 & 0 & & & $0-0.3$ & & \\
\hline Hoplosternum littorale & $0-9.1$ & $0-0.7$ & 0.2 & $0-0.8$ & & & $0-0.7$ & & \\
\hline Hypostomus regani & $0-1.4$ & $0-0.5$ & 7.4 & $0-0.1$ & $0-6.6$ & $0-1.7$ & $0-5.8$ & $0-1.1$ & \\
\hline Characidium zebra & 0 & $0-0.1$ & 0 & & & & $0-0.6$ & & $0-0.2$ \\
\hline Synbranchus cf. marmoratus & $0-0.1$ & $0-0.1$ & 0 & & & & $0-0.4$ & & $0-0.1$ \\
\hline 'Cheirodon'stenodon & $0-48.5$ & $0-1.4$ & & 0 & & & $0-0.1$ & & \\
\hline Leporinus striatus & $0-0.2$ & & 0 & 0 & 0 & 0 & $0-0.5$ & $0-0.2$ & $0-0.1$ \\
\hline Piabarchus stramineus & $0-0 ; 6$ & & 0 & $0-0.5$ & $0-1.3$ & $0-4.0$ & $0-0.7$ & $0-47.9$ & $0-50.0$ \\
\hline Eigenmannia virescens & $0-0.1$ & & 1.0 & 0 & 0 & $0-0.1$ & $0-0.7$ & $0-0.9$ & $0-0.1$ \\
\hline Astyanax bockmanni & $0-0.2$ & & 0.1 & $0-0.2$ & $0-0.2$ & & $0-5.5$ & & \\
\hline Oligosarcus paranensis & $0-1.4$ & & 0.3 & 0 & & & $0-1.0$ & & \\
\hline Hypostomus paulinus & $0-0.2$ & & 0.1 & 0 & & & & & \\
\hline Hemigrammus marginatus & 0 - 0.1 & & $\mathbf{0}$ & & 0 - 43.9 & 0 - 72.9 & $0-2.7$ & $0-0.1$ & $0-54.8$ \\
\hline Rhinodoras dorbignyi & $0-1.4$ & & 0 & & 0 & $0-0.4$ & $0-0.9$ & $0-0.3$ & $0-5.6$ \\
\hline Gymnotus sylvius & $0-0.3$ & & 0 & & & & $0-0.1$ & & \\
\hline Apareiodon piracicabae & $0-1.1$ & & & $0-4.1$ & $0-0.9$ & & $0-3.9$ & $0.2-0.3$ & $0-0.3$ \\
\hline Bryconamericus iheringhii & $0-0.4$ & & & 0 & & & $0-0.2$ & & \\
\hline Leporellus vitatus & $0-0.1$ & & & & $0-0.4$ & 0 & $0-0.9$ & $0-0.3$ & \\
\hline Schizodon intermedius & $0-5.2$ & & & & $0-0.1$ & $0-0.1$ & $0-12.8$ & $0-2.1$ & \\
\hline Acestrorhynchus lacustris & & $1.6-6.5$ & 6.9 & $6.6-17.6$ & $0-9.3$ & $0-1.7$ & 0 - 9.4 & $1.7-4.2$ & $0.1-4.7$ \\
\hline Myloplus tiete & & $0-0.1$ & 0 & $0-0.1$ & 0 & $0-0.1$ & $0-0.3$ & $0-0.4$ & $0-0.1$ \\
\hline Crenicichla britskii & & $0-0.7$ & 0.1 & $0-0.6$ & $0-0.2$ & $0-0.6$ & $0-0.7$ & $0-0.4$ & $0-1.8$ \\
\hline Crenicichla cf. niederleinii & & $0-2.8$ & 0.1 & $0.1-0.2$ & $0-1.1$ & $0-2.1$ & $0-1.3$ & $0-0.3$ & $0-0.1$ \\
\hline Megalancistrus parananus & & $0-0.1$ & 0.7 & 0 & $0-0.4$ & $0-0.2$ & $0-0.1$ & $0.1-0.1$ & $0-0.1$ \\
\hline Hypostomus maragaritifer & & $0-0.1$ & 2.0 & $0-0.1$ & $0-8.2$ & $0-1.6$ & $0-0.4$ & $0-1.4$ & \\
\hline Proloricaria prolixa & & $0-0.1$ & 0.3 & $0.8-2.0$ & $0-1.8$ & $0-7.9$ & $0-0.5$ & $0-0.4$ & \\
\hline Sternopygus macrurus & & $0-0.3$ & 0 & $0.2-0.6$ & & & $0-0.3$ & $0-0.2$ & $0-0.4$ \\
\hline
\end{tabular}


Tab. 4. (continued).

\begin{tabular}{|c|c|c|c|c|c|c|c|c|c|}
\hline Species & JU & $\mathrm{CH}$ & $\mathrm{OU}$ & SG & CA-II & CA-I & $\mathrm{CP}$ & TA & RO \\
\hline Cichlasoma paranaense & & $0-0.1$ & 0.2 & $0-0.1$ & & & $0-0.2$ & & $0-0.9$ \\
\hline Crenicichla haroldoi & & $0-2.9$ & & $0.2-0.2$ & $0-0.2$ & $0-1.3$ & $0-1.2$ & $0-1.2$ & $0-0.2$ \\
\hline Hypostomus iheringii & & 0 & & $0-0.3$ & & & $0-3.2$ & & \\
\hline Crenicichla jaguarensis & & $0-0.3$ & & $0-0.1$ & & & & & \\
\hline Astyanax paranae & & $0-0.1$ & & & & & $0-20.8$ & & \\
\hline Hypostomus hermanni & & & 0 & 0 & 0 & & 0 & & \\
\hline Hypostomus nigromaculatus & & & 0 & 0 & $0-0.2$ & & $0-0.1$ & & \\
\hline Pimelodella gracilis & & & 0 & 0 & & & & & $0-0.1$ \\
\hline Hypostomus albopunctatus & & & 0.2 & & & 0 & $0-1.9$ & & \\
\hline Parodon nasus & & & 0 & & & & $0-1.5$ & & \\
\hline Schizodon altoparanae & & & & 0 & & 0 & $0-0.1$ & $0-0.1$ & $0-0.1$ \\
\hline Leporinus lacustris & & & & 0 & & & $0-0.5$ & $0-0.1$ & $0-7.7$ \\
\hline Apteronotus ellisi & & & & 0 & & & $0-0.5$ & $0-0.1$ & \\
\hline Ageneiosus militaris & & & & $0-0.1$ & & & $0-0.2$ & & $0-0.6$ \\
\hline Hypostomus tietensis & & & & 0 & & & $0-0.3$ & & \\
\hline Callichthys callichthys & $0-0.5$ & & & & & & 0 & & \\
\hline Pimelodella meeki & $0-0.1$ & & & & & & 0 & & \\
\hline Leporellus pictus & 0 & & & & & & & & \\
\hline Hyphessobrycon anisitsi & $0-6.9$ & & & & & & & & \\
\hline Characidium fasciatum & $0-0.4$ & & & & & & & & \\
\hline Phenacorhamdia tenebrosa & & & 0 & & & & & & \\
\hline Planaltina bristkii & & & & 0 & & & & & \\
\hline Corydoras aeneus & & & & 0 & & & & & \\
\hline Hypostomus topavae & & & & $0-0.1$ & & & & & \\
\hline Apareiodon ibitiensis & & & & & 0 & & 0 & & \\
\hline Apteronotus brasiliensis & & & & & $0-0.2$ & & $0-0.1$ & & \\
\hline Pimelodus paranensis & & & & & & 0 & $0-0.1$ & & \\
\hline Megalonema platanum & & & & & & & $0-0.5$ & $0-0.1$ & \\
\hline Pimelodus absconditus & & & & & & & $0-0.1$ & $0-0.9$ & \\
\hline Aphyocharax anisitsi & & & & & & & $0-2.0$ & & $0-5.0$ \\
\hline Brycon nattereri & & & & & & & $0-0.7$ & & \\
\hline Corydoras ehrhardti & & & & & & & $0-0.3$ & & \\
\hline Cetopsis gobioides & & & & & & & $0-0.1$ & & \\
\hline Imparfinis schubarti & & & & & & & $0-0.1$ & & \\
\hline Loricaria simillima & & & & & & & 0 & & \\
\hline Pimelodus microstoma & & & & & & & $0-1.8$ & & \\
\hline Pseudopimelodus mangurus & & & & & & & $0-0.2$ & & \\
\hline Trichomycterus diabolus & & & & & & & $0-0.5$ & & \\
\hline Myloplus levis & & & & & & & & & $0-0.1$ \\
\hline
\end{tabular}


Tab. 5. Distribution of long-distance migratory species in the cascade of reservoirs along the Paranapanema River (gray $=$ presence), based on all studies (47 samples). Numbers are the relative abundance range (percentage, min-max), based on studies that provided abundance data (32 samples); the most abundant species in the cascade are highlighted in bold. Reservoir names (codes) are shown in Fig. 3.

\begin{tabular}{|c|c|c|c|c|c|c|c|c|c|}
\hline Species & $\mathrm{JU}$ & $\mathrm{CH}$ & $\mathrm{OU}$ & SG & CA-II & CA-I & $\mathrm{CP}$ & $\mathrm{TA}$ & RO \\
\hline Megaleporinus obtusidens & $0-1.3$ & $0-0.3$ & 0.1 & $0-0.2$ & $0-1.8$ & $0-1.2$ & $0.2-3.9$ & $0.1-0.5$ & $0-0.6$ \\
\hline Pimelodus maculatus & $0-7.9$ & $3-4.3$ & 2.5 & $1.5-7.5$ & $0-13.2$ & $0-31.8$ & $0.4-5.8$ & $2.4-5.0$ & $0-2.3$ \\
\hline Prochilodus lineatus & $0-0.8$ & $0-0.9$ & 0.1 & 0 & $0-5.3$ & $0-1.6$ & $0-6.4$ & $0.3-0.9$ & \\
\hline Salminus hilarii & $0-0.5$ & & 0.1 & 0 & 0 & 0 & $0-0.8$ & $0-0.1$ & \\
\hline Megaleporinus elongatus & $0-1.1$ & & & 0 & $0-6.1$ & $0-2.5$ & $0-2.8$ & $0-0.3$ & $0-1.0$ \\
\hline Piaractus mesopotamicus & $0-0.1$ & & & 0 & 0 & 0 & $0-0.1$ & & \\
\hline Steindachneridion scriptum & & & 0.1 & $0-0.1$ & 0 & & $0-0.3$ & & \\
\hline Rhinelepis aspera & & & 0.4 & & 0 & $0-0.6$ & $0-0.1$ & $0-0.7$ & $0-0.1$ \\
\hline Salminus brasiliensis & & & & & $0-0.2$ & $0-0.8$ & $0-0.2$ & $0-0.1$ & $0-0.1$ \\
\hline Pinirampus pirinampu & & & & & $0-0.9$ & $0-1.0$ & $0-0.9$ & $0.3-0.8$ & $0-0.4$ \\
\hline Pseudoplatystoma corruscans & & & & & 0 & 0 & $0-0.1$ & & \\
\hline Rhaphiodon vulpinus & & & & & & & $0-1.3$ & $0-0.1$ & $0-5.1$ \\
\hline Zungaro jahu & & & & & & & & $0-0.2$ & \\
\hline Brycon orbignyanus & & & & & & & & & $0-0.08$ \\
\hline
\end{tabular}

Tab. 6. Distribution of non-native species in the cascade of reservoirs along the Paranapanema River (gray= presence), based on all studies (47 samples). Numbers are the relative abundance range (percentage, min-max), based on studies that provided abundance data (32 samples); the most abundant species in the cascade are highlighted in bold. Reservoir names (codes) are shown in Fig. 3.

\begin{tabular}{|c|c|c|c|c|c|c|c|c|c|}
\hline Species & $\mathrm{JU}$ & $\mathrm{CH}$ & $\mathrm{OU}$ & SG & CA-II & CA-I & $\mathrm{CP}$ & TA & $\mathrm{RO}$ \\
\hline Hyphessobrycon eques & $0-0.1$ & & $\mathbf{0}$ & $\mathbf{0}$ & $0-14.3$ & $0-3.6$ & $0-3.2$ & & $0-26.3$ \\
\hline Coptodon rendalli & $0-1.0$ & & 0 & & & & $0-0.3$ & $0-0.7$ & \\
\hline Metynnis lippincottianus & $0-0.3$ & & & $1.0-1.4$ & $0.1-0.2$ & $0.1-4.3$ & $0-3.7$ & $0.1-1.1$ & $0-8.8$ \\
\hline Cichla kelberi & $0-0.7$ & $0-0.8$ & 0.1 & $0-0.4$ & & & $0-5.5$ & & $0-4.3$ \\
\hline Plagioscion squamosissimus & & $2.9-7.6$ & 1.9 & $1.6-1.7$ & 0 - 11.8 & $0-10.0$ & $0-9.4$ & $3.3-8.7$ & 0 - 37.9 \\
\hline Oreochromis niloticus & & $0-11.4$ & & $0-0.1$ & $0-1.1$ & $0-0.1$ & 0 & & $0-0.1$ \\
\hline Hypostomus auroguttatus & & $0-0.4$ & & $0-0.3$ & $0-0.2$ & & & $0-0.3$ & \\
\hline Serrasalmus marginatus & & $0-0.1$ & & $0-0.2$ & & $0-0.2$ & 0 & $0.4-5.1$ & $0-15.5$ \\
\hline Triportheus angulatus & & $0-6.5$ & & 0 & & 0 & $0-1.7$ & $0-0.1$ & \\
\hline Steindachnerina brevipinna & & $0-1.6$ & & $0-1.2$ & & & $0-2.1$ & $0-0.2$ & $0-2.0$ \\
\hline Pimelodella avanhandavae & & $0-0.2$ & & $0-0.1$ & & & $0-0.1$ & & \\
\hline Astronotus crassipinnis & & & 0 & $0.2-0.2$ & & & 0 & & \\
\hline Schizodon borelli & & & & 0 & $0-0.2$ & $0-0.4$ & $0-1.8$ & $0.3-0.6$ & $0-12.0$ \\
\hline Odontostilbe weitzmani & $0-1.3$ & & & & & & & & \\
\hline Hoplias intermedius & 0 & & & & & & & & \\
\hline Hoplias lacerdae & 0 & & & & & & & & \\
\hline Gymnotus inaequilabiatus & $0-0.4$ & & & & & & & & \\
\hline Triportheus nematurus & $0-0.1$ & $0-0.6$ & & & & & & & \\
\hline Cyprinus carpio & 0 & & & 0 & & & & & \\
\hline Hypostomus ternetzi & & $0-0.2$ & 0.1 & & & & & & \\
\hline Cichla piquiti & & $0-0.2$ & & $0-0.1$ & & & & & \\
\hline Poecilia reticulata & & & 0 & & & & & & \\
\hline Hypostomus roseopunctatus & & & 0 & & & & & & \\
\hline Brachyhypopomus gauderio & & & & 0 & & & & & \\
\hline Megaleporinus macrocephalus & & & & $0-0.1$ & 0 & 0 & & & \\
\hline Aphyocharax dentatus & & & & & $0-0.9$ & & & $0-0.1$ & \\
\hline Pseudoplatystoma fasciatum & & & & & & 0 & & & \\
\hline
\end{tabular}


Tab. 6. (continued).

\begin{tabular}{|c|c|c|c|c|c|c|c|c|c|}
\hline Species & $\mathrm{JU}$ & $\mathrm{CH}$ & OU & SG & CA-II & CA-I & $\mathrm{CP}$ & TA & RO \\
\hline Apteronotus cf. caudimaculosus & & & & & & 0 & $0-0.1$ & & \\
\hline Sorubim lima & & & & & & 0 & $0-0.1$ & $0-0.1$ & \\
\hline Tatia intermedia & & & & & & & $0-0.1$ & & \\
\hline Corydoras paleatus & & & & & & & $0-0.1$ & & \\
\hline Hypostomus myersi & & & & & & & $0-0.1$ & & \\
\hline Hypostomus variostictus & & & & & & & $0-0.1$ & & \\
\hline \multicolumn{10}{|l|}{ Pterygoplichthys ambrosetti } \\
\hline Loricariichthys platymetopon & & & & & & & $0-11.6$ & $0.6-13.7$ & $0-13.8$ \\
\hline Clarias gariepinnus & & & & & & & & $0-0.1$ & \\
\hline Roeboides descalvadensis & & & & & & & & $0.1-1.1$ & $0-26.6$ \\
\hline Rhamphichthys hahni & & & & & & & & $0-0.1$ & $0-0.4$ \\
\hline Auchenipterus osteomystax & & & & & & & & $6.2-8.8$ & $0-9.3$ \\
\hline Trachelyopterus galeatus & & & & & & & & $0.7-4.4$ & $0-2.2$ \\
\hline Pterodoras granulosus & & & & & & & & $0-0.3$ & $0-0.3$ \\
\hline Trachydoras paraguayensis & & & & & & & & $0-3.7$ & $0-0.1$ \\
\hline Pimelodus ornatus & & & & & & & & $0-0.1$ & $0-0.5$ \\
\hline Ossancora eigenmanni & & & & & & & & & $0-0.4$ \\
\hline Paraloricaria vetula & & & & & & & & & $0-12.7$ \\
\hline Loricariichthys labialis & & & & & & & & & $0-0.1$ \\
\hline Hypophthalmus edentatus & & & & & & & & & $0-64.7$ \\
\hline Catathyridium jenynsii & & & & & & & & & $0-1.8$ \\
\hline Potamotrygon cf. motoro & & & & & & & & & $0-0.1$ \\
\hline Satanoperca aff. pappaterra & & & & & & & & & $0-7.2$ \\
\hline
\end{tabular}

\section{Discussion}

Our study is the first to compile data on fish diversity in the cascade of reservoirs along the Paranapanema River. This compilation, based on studies conducted in the last decades (1990 to 2012), revealed the presence of 161 species, being 111 native and 50 non-native. The accumulation curve indicated that most native species in the reservoirs were recorded, and we estimated total richness to range between 125 and 132 species. Previous estimates indicated 155 fish species in the Paranapanema river basin (Duke Energy, 2008), and 270 to 310 in the upper Paraná river basin (Agostinho et al., 2007b; Langeani et al., 2007). Impoundments in the Paranapanema River, therefore, hold a high number of fish species and contribute significantly to the basin species pool; a number that may increase if other environments are sampled (i.e., downstream segments, streams, free-flowing stretches). We are aware that misidentifications, re-descriptions and taxonomic revisions may add uncertainty to estimates in impoundments, and we followed recent literature to avoid or minimize nomenclatural inconsistencies. Future studies may refine species lists and distribution ranges, including wholebasin monitoring programs, with standardized protocols and multi-gear sampling strategies.

The number of native species varied among reservoirs in the cascade system (range: 45 to 92), but most impoundments had similar values ( $c a .50$ species). Nonmigratory fishes dominated assemblages, confirming our prediction and previous studies (e.g., Agostinho et al., 2007a). These species prevailed in richness, abundance and occurrence, especially small and medium-sized characids, a common pattern in Neotropical reservoirs (Barrella, Petrere Jr., 2003; Luiz et al., 2005; Agostinho et al., 2016). These values are higher than those reported by Agostinho et al. (2007a), which found between 20 and 40 species in Brazilian reservoirs. However, Agostinho et al. (2007a) estimates were based on gillnets, while studies gathered here used different sampling gears. In addition, some reservoirs were sampled multiple times over a considerable period (e.g., Jurumirim, Salto Grande, Capivara, Rosana). We highlight also that impoundments in the Paranapanema River provide relevant habitats for small-sized species, such as submerged trees, aquatic macrophytes and lateral lagoons (e.g., Casatti et al., 2003; Pelicice et al., 2005; Ferrareze, Nogueira, 2011). In particular, three reservoirs (i.e., Ourinhos, Salto Grande and Capivara) were more species rich, but different mechanisms may be involved. Ourinhos (60 species), for example, was evaluated soon after its filling; in this stage, fishes are disoriented and populations are randomly distributed, with weak environmental selection. Previous studies have reported increases in species richness in the first years after river regulation, with declines in subsequent years (Agostinho et al., 2007a). Salto Grande (67 species), on the other hand, is a small and old impoundment sampled with a variety of gears, what may result in surveys that are more complete. In addition, environmental heterogeneity 
may have played a role, considering that Salto Grande has relevant habitat diversity (Brandão et al., 2009). This mechanism seems to be even more important in Capirava (80 species), which preserves remnant lotic areas and large tributaries (Hoffmann et al., 2005; Pelicice, Agostinho, 2008; Bennemann et al., 2013). The remaining reservoirs showed similar values of species richness ( $c a .50$ species), although we expected increasing values along the cascade system (e.g., Oberdorff et al., 1993; Araújo et al., 2009; Foubert et al., 2018). The convergence of species richness among impoundments (see also Agostinho et al., 2007a) is probably linked to the loss of fluvial heterogeneity along the main channel, together with the creation of similar and homogeneous habitats (i.e., impoundments). Neotropical fishes are primarily rheophilic, demanding specific habitats and flow conditions to survive and reproduce; few species evolved traits to cope with lentic habitats (Gomes, Miranda, 2001). Declines in species richness is a common pattern in rivers impacted by dams (e.g., Barrella, Petrere Jr., 2003; Agostinho et al., 2007a; Hoeinghaus et al., 2009; Petesse, Petrere Jr., 2012; Araújo et al., 2013). Long-term studies, in particular, have shown that fish assemblages in impounded areas are substantially different from the structure found in pristine conditions (Mol et al., 2007; Orsi, Britton, 2014; Lima et al., 2016). Future studies, therefore, must improve our understanding about factors that determine species richness in Neotropical reservoirs, as a means of improving predictions about diversity losses in new impoundments.

While most reservoirs were similar in respect to species richness, the composition of native non-migratory fishes changed considerably along the cascade. Some species were widely distributed, but each reservoir tended to have specific composition. Variation in fish composition along longitudinal gradients is a common pattern in large river systems (e.g., Araújo et al., 2009; Foubert et al., 2018), usually in response to spatial and environmental components. Species are deleted/added to local assemblages, creating turnover and/or nestedness patterns along the river corridor. Impoundments, however, add discontinuity to the river continuum (Ward, Stanford, 1983) and provide common habitats irrespective to their position in the basin, usually associated with lentic conditions, shallow shores, vast water column and deep sites. These new environmental conditions select common traits and homogenize fish diversity in functional terms (Santos et al., 2017). In this sense, reservoirs promote similar pressures upon the fish fauna and favor opportunistic or lentic-adapted species from the local species pool, forming simplified versions of the original riverine community. However, species are assembled from different species pools along the cascade system, resulting in divergent species composition among reservoirs. Therefore, even though species richness and functional traits are convergent among impoundments, taxonomical composition is divergent (i.e., beta diversity), explaining the high species richness observed in the cascade system. However, the sequence of impoundments must have changed beta diversity patterns that characterize riverine networks (e.g., Vitorino Jr. et al., 2016), probably increasing the similarity among reservoirs (e.g., Petesse, Petrere Jr., 2012). Data collected prior to river regulation is crucial to understand community assembling processes (e.g., Araújo et al., 2013; Lima et al., 2016), but this type of data is rare in the Paranapanema basin.

As expected, migratory fishes showed low species richness and abundance in the cascade system. Although we recorded the presence of 14 species, especially in lower reaches, the abundance of migratory fishes was very low in all impoundments. The decline/collapse of migratory species is recurrent in regulated rivers (Petrere Jr., 1996; Okada et al., 2005; Godinho, Kynard, 2008; Pelicice, Agostinho, 2008), since these fishes demand different fluvial habitats for feeding and reproducing, migrating over variable distances to complete their life cycle (Pompeu et al., 2012). The cascade of dams along the Paranapanema River prevents long-distance migration and creates environments adverse to migratory fishes (i.e., lentic areas, flow regulation). Pimelodus maculatus was the most important, but it also summed low abundance in most samples. This catfish is usually common in reservoirs of the upper Paraná basin, probably because it is small-sized, migrates over shorter distances and displays opportunistic feeding behavior (Lima-Junior, Gointein, 2004; Paschoalini et al., 2013). Large-sized species, in particular, were recorded only downstream Salto Grande dam. This pattern may be related to biogeographic factors, since waterfalls were present in the area before dam construction, constraining fish movements. However, the position of reservoirs in the cascade must also be considered to explain the distribution of migratory fishes. The upper/middle reaches (between Jurumirim and Canoas I) are the most affected by river regulation, because 8 dams are distributed over $200 \mathrm{~km}$. This sequence of impoundments creates sections where lotic segments are short or absent, with no critical habitat for migratory and rheophilic fishes (e.g., lateral lagoons, backwaters, free-flowing stretches, tributaries). The area with greatest potential to support migratory fish populations is the upper section of the Capivara reservoir, which preserves large tributaries (Hoffmann et al., 2005; Vianna, Nogueira, 2008; Bennemann et al., 2013), especially the Tibagi river (Bennemann et al., 2000; Shibatta et al., 2007). This impoundment showed the highest species richness, including virtually all migratory species of the basin. Early life forms of large-size species have been recorded in the area, indicating the presence of spawning areas and nursery grounds (Orsi et al., 2016). However, the construction of fish ladders at Canoas I and II dams affected the distribution of migratory fishes. Populations that recruited in the area of Capivara passed upstream and remained confined in short sections between Canoas I and Salto Grande dam (Lopes et al., 2007; Pelicice, Agostinho, 2008; Britto, Carvalho, 2013). The greater abundance of migratory species in Canoas I and II indeed suggest that fishes migrated upstream and evaded Capivara reservoir soon after the installation of ladders, as indicated previously 
(Pelicice, Agostinho, 2008; Britto, Carvalho, 2013). Another region with potential to maintain migratory fishes is the upper section of Rosana reservoir, which has a short lotic stretch with fluvial lagoons. However, Rosana dam is located $c a .25 \mathrm{~km}$ from the confluence with the Paraná river; this dam blocked upward fish movements and isolated the Paranapanema basin (Antonio et al., 2007). In addition, Ferrareze, Nogueira (2011) captured no young migratory fish in lateral lagoons, possibly due to the lack of spawning grounds upstream. Similarly, Kipper et al. (2011) reported very low abundance of larvae across the reservoir, indicating that recruitment is negligible. Finally, large scale monitoring did not record eggs and larvae of important large-size species (e.g., Piaractus mesopotamicus, Prochilodus lineatus) in this reservoir (Orsi et al., 2016). Therefore, migratory fish populations in reservoirs along the Paranapanema River are significantly disturbed, probably with low or no recruitment in many sections and impoundments, particularly in middle and upper reaches. The maintenance of migratory fish populations, much appreciated in riverine and floodplain fisheries, will depend on the preservation of lotic remnants and fluvial habitats (e.g., Vianna, Nogueira, 2008; Suzuki et al., 2013; Barzotto et al., 2015), together with the revision of current management practices (i.e., Pompeu et al., 2012). In this sense, the area affected by Capivara reservoir should receive conservation efforts, particularly to maintain ecosystem functionality in tributaries (e.g., Tibagi, Cinzas).

Another important result is the high number of non-native species in the impoundments (50 species), which accounted for $30.5 \%$ of total richness. This is an impressive high number, confirmed by another recent study that reported 47 non-native species in the basin (Garcia et al., 2018). This scenario is not an exception, considering that the upper Paraná river basin is highly invaded by multiple non-native species (e.g., Britton, Orsi, 2012; Vitule et al., 2012; Orsi, Britton, 2014; Ortega et al., 2015). Our data showed that some species have wide distribution (e.g., P. squamosissimus, H. eques, O. niloticus, S. marginatus, Schizodon borelli, Metynnis lippincottianus, C. kelberi), explaining why most reservoirs were similar in composition. However, many species were restricted to one or a few reservoirs, probably because of sporadic introductions that occurred in specific impoundments. This trend created a compositional turnover along the cascade, increasing the richness of non-native species at the basin level. In general, introductions occur due to different reasons (Ortega et al., 2015; Azevedo-Santos et al., 2015), but aquaculture, fish stocking, sport fishing, aquarium dumping, and the removal of natural barriers probably caused massive fish introductions in the Paranapanema River (Júlio Jr. et al., 2009; Agostinho et al., 2010; Britton, Orsi, 2012; Garcia et al., 2018). These actions caused the release of species with high invasive risk, which are currently widespread (e.g., O. niloticus, Coptodon rendalli, C. kelberi, $S$. marginatus, $P$. squamosissimus). It is not possible to confirm that all 50 species are established in these impoundments (i.e., recruiting), but the presence of early life forms collected in different localities (Almeida et al., 2018) indicate that some are reproducing. Furthermore, non-native species were abundant in most samples, summing relevant fraction or dominating local assemblages. Reservoirs located downstream were more invaded (i.e., species richness and abundance), supporting previous studies that reported high invasion rates (Garcia et al., 2018) or strong impacts (Orsi, Britton, 2014; Pelicice, Agostinho, 2009). Jurumirim reservoir was the less invaded and, consequently, has been considered a model for sustainable artisanal fisheries (Novaes, Carvalho, 2013), because fishing activities are based on native species, with higher prices, and may be sustained in the long term. The current scenario in the cascade of impoundments, therefore, demands immediate management actions to control populations (e.g., selective fishing) and to prevent new introductions (e.g., education, inspection), mainly because impoundments are vulnerable to new introductions (e.g., Espinola et al., 2010; Franco et al., 2018). Non-native species impact native biodiversity in multiple ways, causing population declines, extirpation or homogenization, as recorded by studies conducted in the Paraná river basin (e.g., Pelicice, Agostinho, 2009; Vitule et al., 2012; Orsi, Britton, 2014; Daga et al., 2015) and elsewhere (Cucherousset, Olden, 2011; Azevedo-Santos et al., 2017). Equally important is the implementation of long-term monitoring in the cascade system, to perform robust studies about impacts, invasion dynamics (e.g., establishment, invasion) and broad surveys. The difference in total richness between our study and Garcia et al. (2018), for example, suggests that the real number of non-native species is uncertain. Updated surveys are, therefore, crucial to understand biological invasions and minimize negative impacts.

In general, our results confirm trends reported in other regulated rivers (Petrere Jr. 1996; Barrella, Petrere Jr. 2003; Agostinho et al., 2007b; Daga, Gubiani, 2012; Petesse, Petrere Jr., 2012; Agostinho et al., 2016), where the diversity of migratory fishes is low and non-native species are common. The present study cannot infer about demographic trends and long-term dynamics, since our data come from multiple studies that applied different sampling methodologies. However, fish assemblage structure is probably stable in the studied reservoirs, especially because most are old, constructed decades ago. Specific environmental filters created by the impoundment constrain fish assemblages to persist in another state (Agostinho et al., 2016; Santos et al., 2017). In this sense, the current flow regime in the Paranapanema river, ruled by the cascade of dams, is the main force behind ecosystem state and dynamics, determining patterns of diversity and productivity. Conservation plans must consider this scenario to set appropriate management actions, especially because a number of studies have questioned the efficacy of current practices, i.e., fish passages and stocking (Pelicice, Agostinho, 2008; Agostinho et al., 2010; Pelicice et al., 2015a; Lira et al., 2017). Fish passages in Canoas dams, in particular, caused significant impacts 
on fish populations (Pelicice, Agostinho, 2008; Casimiro et al., 2017), declining the conservation status of migratory fishes. In addition, stocking practices, conducted for decades (Agostinho et al., 2010), contributed to introduce several non-native species (Britton, Orsi, 2012; Ortega et al., 2015; Garcia et al., 2018), but was unable to restore populations of migratory fishes in impoundments. This situation should concern authorities about the conservation of Neotropical fishes in light of severe human disturbances (Pelicice et al., 2017), because rivers are extensively regulated, while the watershed is subjected to extensive agriculture and urban development, with activities that promote land cover changes and use non-native species (e.g., aquaculture). Conservation will depend on measures that preserve fluvial remnants (e.g., tributaries, such as the Tibagi and Cinza rivers), regulate human activities in surrounding areas, curb the introduction of non-native species, and control/ eradicate populations already introduced (see Bennemann et al., 2000; Britton, Orsi, 2012; Orsi et al., 2016; Pelicice et al., 2017). We emphasize that native fishes in these impoundments must receive conservation efforts, since they represent a relevant fraction of regional diversity, in addition to sustaining ecosystem services that are valued by artisanal and recreational fisheries.

\section{Acknowledgments}

The authors thank their respective institutions. AAA received a CNPq productivity grant, and VMAS received a CAPES scholarship. We are grateful to the Editor and reviewers who provided valuable comments and corrections. We also thank Geovana S. Andrade (UFT) for helping with data organization.

\section{References}

Abilhôa V, Bastos LP. Composição e estrutura da ictiofauna da Estação Ecológica do Caiuá, área de influência da UHE de Rosana (rio Paranapanema), sudeste do Brasil. Arq Ciênc Vet Zool UNIPAR. 2005; 8(1):33-9.

Agostinho AA, Gomes LC, Suzuki HI, Júlio Jr HF. Migratory fish from the upper Parana river basin, Brazil. In: Carolsfeld J, Harvey B, Ross C, Baer A, editors. Migratory fishes of South America: biology, fisheries and conservation status. Victoria: World Fisheries Trust, the World Bank and the International Development Research Centre; 2003. p.19-99.

Agostinho AA, Gomes LC, Pelicice FM. Ecologia e Manejo de Recursos Pesqueiros em Reservatórios do Brasil. Maringá: Eduem; 2007a.

Agostinho AA, Pelicice FM, Petry AC, Gomes LC, Júlio Júnior HF. Fish diversity in the upper Paraná River basin: habitats, fisheries, management and conservation. Aquat Ecosyst Health Manag. 2007b; 10(2):174-86.

Agostinho AA, Pelicice FM, Gomes LC, Júlio Júnior HF. Reservoir fish stocking: when one plus one may be less than two. Nat Conservação. 2010; 8(2):103-11.
Agostinho AA, Gomes LC, Santos NCL, Ortega JCG, Pelicice FM. Fish assemblages in Neotropical reservoirs: colonization patterns, impacts and management. Fish Res. 2016; 173(1):26-36.

Almeida FS, Frantine-Silva W, Lima SC, Garcia DAZ, Orsi ML. DNA barcoding as a useful tool for identifying non-native species of freshwater ichthyoplankton in the neotropics. Hydrobiologia. 2018; 817(1):111-19.

Antonio RR, Agostinho AA, Pelicice FM, Bailly D, Okada EK, Dias JHP. Blockage of migration routes by dam construction: can migratory fish find alternative routes? Neotrop Ichthyol. 2007; 5(2):177-84. Available from: http://dx.doi.org/10.1590/ S1679-62252007000200012

Araújo ES, Marques EE, Freitas IS, Neuberger AL, Fernandes R, Pelicice FM. Changes in distance decay relationships after river regulation: similarity among fish assemblages in a large Amazonian river. Ecol Fresh Fish. 2013; 22(4):543-52.

Araújo FG, Pinto BCT, Teixeira TP. Longitudinal patterns of fish assemblages in a large tropical river in southeastern Brazil: Evaluating environmental influences and some concepts in river ecology. Hydrobiologia. 2009; 618:89-107.

Arcifa MS, Esguícero ALH. The fish fauna in the fish passage at the Ourinhos Dam, Paranapanema River. Neotrop Ichthyol. 2012; 10(4):715-22. Available from: http://dx.doi. org/10.1590/S1679-62252012000400004

Azevedo-Santos VM, Pelicice FM, Lima-Junior DP, Magalhães ALB, Orsi ML, Vitule JRS, Agostinho AA. How to avoid fish introductions in Brazil: education and information as alternatives. Nat Conservação. 2015; 13(2):123-32.

Azevedo-Santos VM, Vitule JRS, Pelicice FM, García-Berthou E, Simberloff D. Nonnative fish to control Aedes mosquitoes: a controversial, harmful tool. BioScience. 2017; 67(1):84-90.

Barrella W, Petrere Jr. M. Fish community alterations due to pollution and damming in Tietê and Paranapanema rivers (Brazil). River Res Appl. 2003; 19(1):59-76.

Barzotto E, Sanches PV, Bialetzki A, Orvati L, Gomes LC. Larvae of migratory fish (Teleostei: Ostariophysi) in the lotic remnant of the Paraná River in Brazil. Zoologia. 2015; 32(4):270-80.

Bennemann ST, Galves W, Capra LG. Recursos alimentares utilizados pelos peixes e estrutura trófica de quatro trechos no reservatório Capivara (Rio Paranapanema). Biota Neotrop. 2013; 11(1):63-71.

Bennemann ST, Shibatta OA, Garavello JC. Peixes do rio Tibagi: uma abordagem ecológica. Londrina: Editora UEL; 2000.

Brandão H, Vidotto-Magnoni AP, Ramos IP, Carvalho ED. Assessment of the ichthyofauna in stretches under the influence of Salto Grande Reservoir (Middle Paranapanema River, SP/PR, Brazil). Acta Limnol Bras. 2009; 21(4):451-63.

Britto SGC. A estratégia reprodutiva dos peixes migradores frente às escadas do complexo Canoas (Rio Paranapanema, Bacia do Alto Paraná). [PhD Thesis]. Botucatu, SP: UNESP; 2009.

Britto SGC, Carvalho ED. Ecological attributes of fish fauna in the Taquaruçu Reservoir, Paranapanema River (Upper Paraná, Brazil): composition and spatial distribution. Acta Limnol Bras. 2006; 18(4):377-88. 
Britto SGC, Carvalho ED. Reproductive migration of fish and movement in a series of reservoirs in the Upper Parana River basin, Brazil. Fisheries Manag Ecol. 2013; 20(5):426-33.

Britto SGC, Sirol RN. Transposição de peixes como forma de manejo: as escadas do Complexo Canoas, Médio Rio Paranapanema, Bacia do Alto Paraná. In: Nogueira MG, Henry R., Jorcin A, editors. Manejo de reservatórios: impactos potenciais, ações de manejo e sistemas em cascata. $2^{\mathrm{a}}$ ed., São Carlos: RiMa Editora; 2007. p.285-304.

Britton JR, Orsi ML. Non-native fish in aquaculture and sport fishing in Brazil: economic benefits versus risks to fish diversity in the upper River Paraná Basin. Rev Fish Biol Fish. 2012; 22(3):555-65.

Caetano DLF, Gatzke EG, Silva LCP, Vieira FEG. Ictiofauna presente no reservatório de Chavantes, município de Ribeirão Claro - PR. In: Anais do XI Congresso de Educação do Norte Pioneiro. 2011. p.361-369.

Carvalho ED, Marcus LR, Foresti F, Silva VFB. Fish assemblage attributes in a small oxbow lake (Upper Paraná River Basin, São Paulo State, Brazil): species composition, diversity and ontogenetic stage. Acta Limnol Bras. 2005; 17(1):45-56.

Carvalho ED, Silva VFB, Fujihara CY, Henry R, Foresti F. Diversity of fish species in the River Paranapanema Jurumirim Reservoir transition region (São Paulo, Brazil). Ital J Zool. 1998; 65(S1):325-30.

Carvalho ED, Silva VFB. Aspectos ecológicos da ictiofauna e da produção pesqueira do reservatório de Jurumirim (Alto do Paranapanema, SP). In: Henry R, editor. Ecologia de reservatórios: estrutura, funções e aspectos sociais. Botucatu: FUNDIBIO, FAPESP; 1999. p.769-800.

Casatti L, Mendes HF, Ferreira KM. Aquatic macrophytes as feeding site for small fishes in the Rosana Reservoir, Paranapanema River, southeastern Brazil. Braz J Biol. 2003; 63(2):213-22.

Casimiro ACR, Garcia DAZ, Costa ADA, Britton JR, Orsi, ML. Impoundments facilitate a biological invasion: Dispersal and establishment of non-native armoured catfish Loricariichthys platymetopon (Isbrückler \& Nijssen, 1979) in a Neotropical river. Limnologica. 2017; 62:34-37.

CBA (Companhia Brasileira de Alumínio). Relatório de Consolidação das Campanhas de Monitoramento da Ictiofauna, na Fase Pós-Enchimento, do Reservatório da UHE Ourinhos da Companhia Brasileira de Alumínio; 2009.

Colwell RK. EstimateS: statistical estimation of species richness and shared species from samples (software and user's guide) [Internet]. Version 8; 2006 [cited 2016 May 25]. Available from: purl.oclc.org/estimates

Cucherousset J, Olden JD. Ecological impacts of non-native freshwater fishes. Fisheries. 2011; 36(5):215-30.

Daga VS, Gubiani EA. Variations in the endemic fish assemblage of a global freshwater ecoregion: associations with introduced species in cascading reservoirs. Acta Oecol. 2012; 41:95-105.

Daga VS, Skóra F, Padial AA, Abilhoa V, Gubiani ÉA, Vitule JRS. Homogenization dynamics of the fish assemblages in Neotropical reservoirs: comparing the roles of introduced species and their vectors. Hydrobiologia. 2015; 746(1):327-47.
Dias JHP. Distribuição espacial e temporal da ictiofauna do trecho médio do rio Paranapanema e suas relações com as características morfométricas e limnológicas dos compartimentos da bacia. [PhD Thesis]. São Carlos, SP: UFSCar; 2003.

Dias JHP, Garavello JC. Ecological studies on the fish community of Salto Grande Reservoir, Paranapanema River Basin, São Paulo State, Brazil. Verh Internat Verein Limnol. 1998; 26(5):2228-31.

Duke Energy. Peixes do rio Paranapanema. 2nd ed. São Paulo: Horizonte Geográfico; 2008.

Eschmeyer WN, Fong JD. Species by family/subfamily. [Internet]. San Francisco (CA): California Academy of Sciences; 2017. [cited 2018 Mar 01]. Available from: http:// researcharchive.calacademy.org/research/ichthyology/catalog/ SpeciesByFamily.asp

Eschmeyer WN, Fricke R, van der Laan R, editors. Catalog of fishes: genera, species, references. [Internet]. San Francisco (CA): California Academy of Sciences; 2017. [cited 2017 Jul 01]. Available from: http:// researcharchive.calacademy.org/ research/ichthyology/catalog/fishcatmain.asp

Espínola LA, Minte-Vera CV, Júlio Jr. HF. Invasibility of reservoirs in the Paraná Basin, Brazil, to Cichla kelberi Kullander and Ferreira, 2006. Biol Invasions. 2010; 12(6):1873-88.

Ferrareze M, Nogueira MG. Importance of lateral lagoons for the ichthyofauna in a large tropical reservoir. Braz J Biol. 2011; 71(4):807-20.

Foubert A, Lecomte F, Legendre P, Cusson, M. Spatial organisation of fish communities in the St. Lawrence River: a test for longitudinal gradients and spatial heterogeneities in a large river system. Hydrobiologia. 2018; 809(1):155-73.

Franco ACS, Santos LN, Petry AC, García-Berthou E. Abundance of invasive peacock bass increases with water residence time of reservoirs in southeastern Brazil. Hydrobiologia. 2018; 817(1):155-66.

Froese R, Pauly D, editors. FishBase [Internet]. Rome: FAO; 2016. [cited 2017 Jul 01]. Available from: http://fishbase.org

Garavello JC, Britski HA, Zawadzki CH. The cascudos of the genus Hypostomus Lacépède (Ostariophysi: Loricariidae) from the rio Iguaçu basin. Neotrop Ichthyol. 2012; 10(2):263-83. Available from: http://dx.doi.org/10.1590/S1679-62252012000200005

Garcia DAZ, Britton JR, Vidotto-Magnoni AP, Orsi ML. Introductions of non-native fishes into a heavily modified river: rates, patterns and management issues in the Paranapanema River (Upper Paraná ecoregion, Brazil). Biol Invasions. 2018; 20(5):1229-41.

Godinho AL, Kynard B. Migratory fishes of Brazil: life history and fish passage needs. River Res Appl. 2008; 25(6):702-12.

Gomes LC, Miranda LE. Riverine characteristics dictate composition of fish assemblages and limit fisheries in reservoirs of the Upper Paraná River Basin. Regul Rivers. 2001; 17(1):67-76.

Governo do Estado de São Paulo. Sistema Integrado de Gerenciamento de Recursos Hídricos do Estado de São Paulo [Internet]. São Paulo: Governo do Estado de São Paulo; 2018 [cited 2018 May 23]. Available from: http://www.sigrh.sp.gov.br 
Graça WJ, Pavanelli CS. Peixes da planície de inundacão do alto rio Paraná e áreas adjacentes. Maringa: Eduem; 2007.

Hammer $\varnothing$, Harper DAT, Ryan PD. Past: Paleontologia Statistics software package for education and data analysis. Palaeontol Electronica. 2011; 4.

Hoeinghaus DJ, Agostinho AA, Gomes LC, Pelicice FM, Okada EK, Latini JD, Kashiwaqui EAL, Winemiller KO. Effects of river impoundment on ecosystem services of large tropical rivers: embodied energy and market value of artisanal fisheries. Conserv Biol. 2009; 23(5):1222-31.

Hoffmann AC, Orsi ML, Shibatta AO. Diversidade de peixes do reservatório da UHE Escola Engenharia Mackenzie (Capivara), Rio Paranapanema, bacia do alto rio Paraná, Brasil, e a importância dos grandes tributários na sua manutenção. Iheringia Sér Zool. 2005; 95(3):319-25.

Júlio Júnior HF, Dei Tós C, Agostinho AA, Pavanelli CS. A massive invasion of fish species after eliminating a natural barrier in the upper rio Paraná basin. Neotrop Ichthyol. 2009; 7(4):709-18. Available from: http://dx.doi.org/10.1590/S167962252009000400021

Kipper D, Bialetzki A, Santin M. Composição taxonômica da assembleia de larvas de peixes no reservatório de Rosana, Rio Paranapanema, Brasil. Biota Neotrop. 2011; 11(1):421-26.

Kurchevski G, Carvalho ED. Os peixes da represa de Jurumirim: revisão temporal de estudos independentes. In: Henry R, editor. Represa de Jurumirim: ecologia, modelagem e aspectos sociais. Ribeirão Preto: Editora Holos; 2014. p.325-337.

Langeani F, Castro RMC, Oyakawa OT, Shibatta OA, Pavanelli CS, Casatti L. Diversidade da ictiofauna do Alto Rio Paraná: composição atual e perspectivas futuras. Biota Neotrop. 2007; 7(3):181-97.

Lima AC, Agostinho CS, Sayanda D, Pelicice FM, Soares AMVM, Monaghan KA. The rise and fall of fish diversity in a Neotropical river after impoundment. Hydrobiologia. 2016; 763(1):207-21.

Lima-Junior SE, Gointein R. Diet and feeding activity of Pimelodus maculatus (Osteichthyes, Pimelodidae) in the Piracicaba River (State of São Paulo, Brazil) - the effect of seasonality. B Inst Pesca. 2004; 30(2):135-40.

Lira NA, Pompeu PS, Agostinho CS, Agostinho AA, Arcifa MS, Pelicice FM. Fish passages in South America: an overview of studied facilities and research effort. Neotrop Ichthyol. 2017; 15(2):e160139. Available from: http://dx.doi. org/10.1590/1982-0224-20160139

Lopes CM,Almeida FS, Orsi ML, Britto SGC, Sirol RN, Sodré LMK. Fish passage ladders from Canoas Complex - Paranapanema River: evaluation of genetic structure maintenance of Salminus brasiliensis (Teleostei: Characiformes). Neotrop Ichthyol. 2007; 5(2):131-38. Available from: http://dx.doi.org/10.1590/ S1679-62252007000200006

Luiz EA, Petry AC, Pavanelli CS, Júlio Jr HF, Latini JD, Domingues VM. As assembléias de peixes de reservatórios hidrelétricos do Estado do Paraná e bacias limítrofes. In Rodrigues L., Thomas SM, Agostinho AA, Gomes LC, editors. Biocenoses em reservatórios: padrões espaciais e temporais. São Carlos: RiMa; 2005. p.169-184.
Magnoni APV. Ecologia trófica das assembléias de peixes do reservatório de Chavantes (Médio rio Paranapanema, SP/ PR). [PhD Thesis]. Botucatu, SP: UNESP; 2009.

Mol JH, de Mérona B, Ouboter PE, Sahdew S. The fish fauna of Brokopondo Reservoir, Suriname, during 40 years of impoundment. Neotrop Ichthyol. 2007; 5(3):351-68. Available from: http://dx.doi.org/10.1590/S1679-62252007000300015

Novaes JLC, Carvalho ED. Recursos pesqueiros oriundos da pesca artesanal no reservatório de Jurumirim, rio Paranapanema, alto Paraná, Brasil. B Inst Pesca. 2009; 35(4):553-65.

Novaes JLC, Carvalho ED. Analysis of artisanal fisheries in two reservoirs of the upper Paraná River basin (Southeastern Brazil). Neotrop Ichthyol. 2013; 11(2):403-12. Available from: http://dx.doi.org/10.1590/S1679-62252013005000002

Oberdorff T, Guilbert E, Lucchetta JC. Patterns of fish species richness in the Seine River basin, France. Hydrobiologia. 1993; 259(3):157-67.

Okada EK, Agostinho AA, Gomes LC. Spatial and temporal gradients in artisanal fisheries of a large Neotropical reservoir, the Itaipu Reservoir, Brazil. Can J Fish Aquat Sci. 2005; 62(3):714-24.

Orsi ML, Almeida FS, Swarça AC, Claro-García A, Vianna NC, Garcia DAZ, Bialetzki A. Ovos, larvas e juvenis dos peixes da Bacia do Rio Paranapanema uma avaliação para a conservação. Assis, SP: Triunfal Gráfica e Editora, Duke Energy; 2016.

Orsi ML, Britton JR. Long-term changes in the fish assemblage of a Neotropical hydroelectric reservoir. J Fish Biol. 2014; 84(6):1964-70.

Ortega JCG, Júlio Júnior HF, Gomes LC, Agostinho AA. Fish farming as the main driver of fish introductions in Neotropical reservoirs. Hydrobiologia. 2015; 746(1):147-58.

Paschoalini AL, Perini VR, Ribeiro DM, Formagio PS, Rizzo E, Bazzoli N. Reproduction of Pimelodus maculatus (Siluriformes: Pimelodidae) in three section of Grande River basin, downstream Porto Colombia dam, south-eastern Brazil. Neotrop Ichthyol. 2013; 11(3):615-23. Available from: http://dx.doi.org/10.1590/S1679-62252013000300015

Pelicice FM, Agostinho AA, Thomaz SM. Fish assemblages associated with Egeria in a tropical reservoir: investigating the effects of plant biomass and diel period. Acta Oecol. 2005; 27(1):9-16.

Pelicice FM, Agostinho AA. Fish-passage facilities as ecological traps in large Neotropical rivers. Conserv Biol. 2008; 1(22):180-88.

Pelicice FM, Agostinho AA. Fish fauna destruction after the introduction of a non-native predator (Cichla kelberi) in a Neotropical reservoir. Biol Invasions. 2009; 11(8):1789-801.

Pelicice FM, Latini JD, Agostinho AA. Fish fauna disassembly after the introduction of a voracious predator: main drivers and the role of the invader's demography. Hydrobiologia. 2015b; 746(1): 271-83.

Pelicice FM, Pompeu PS, Agostinho AA. Large reservoirs as ecological barriers to downstream movements of Neotropical migratory fish. Fish and Fish. 2015a; 16(4):697-715. 
Pelicice FM, Azevedo-Santos VM, Vitule JRS, Orsi ML, Lima Junior DP, Magalhães ALB, Pompeu PS, Petrere Jr M, Agostinho AA. Neotropical freshwater fishes imperilled by unsustainable policies. Fish and Fish. 2017; 18(6):1119-33.

Petesse ML, Petrere Jr. M. Tendency towards homogenization in fish assemblages in the cascade reservoir system of the Tietê river basin, Brazil. Ecol Eng. 2012; 48:109-16.

Petrere Jr. M. Fisheries in large tropical reservoirs in South America. Lakes Reservoirs. 1996; 2(1-2):111-33.

Poff NL, Allan JD, Bain MB, Karr JR, Prestegaard KL, Richter $\mathrm{BD}$, Sparks RE, Stromberg JC. The natural flow regime. BioScience. 1997; 47(11):769-84.

Pompeu PS, Agostinho AA, Pelicice FM. Existing and future challenges: the concept of successful fish passage in South America. River Res Appl. 2012; 28(4):504-12.

Pringle CM, Freeman MC, Freeman B. Regional effects of hydrologic alterations on riverine macrobiota in the New World: Tropical-Temperate comparisons. Bioscience. 2000; 50(9):807-23.

Reis RE, Kullander SO, Ferraris Jr. CJ. Check list of the freshwater fishes of South and Central America. Porto Alegre: Edipucrs; 2003.

Santos NCL, Santana HS, Ortega JCGA, Dias RM, Stegmann LF, Araújo IMS, Severi W, Bini LM, Gomes LC, Agostinho AA. Environmental filters predict the trait composition of fish communities in reservoir cascades. Hydrobiologia. 2017; 802(1):245-53.

Shibatta OA, Gealh AM, Bennemann ST. Ictiofauna dos trechos alto e médio da bacia do rio Tibagi, Paraná, Brasil. Biota Neotrop. 2007; 7(2):125-34.
Suzuki FM, Zambaldi LP, Pompeu FS. Mapping the critical habitats for migratory species of the upper Grande River region, Minas Gerais State, Brazil. Appl Ecol Env Res. 2013; 11(4):645-59.

Vianna NC. Deplecionamento induzido do reservatório de Salto Grande (Rio Paranapanema, SP/PR: influência sobre a ictiofauna de lagoas marginais. [PhD Thesis]. Botucatu, SP: UNESP; 2008.

Vianna NC, Nogueira MG. Ichthyoplankton and limnological factors in the Cinzas River - an alternative spawning site for fishes in the middle Paranapanema River basin, Brazil. Acta Limnol Bras. 2008; 20(2):139-51.

Vitorino Jr OB, Fernandes R, Agostinho CS, Pelicice FM. Riverine networks constrain B-diversity patterns among fish assemblages in a large Neotropical river. Fresh Biol. 2016; 61(10):1733-45.

Vitule JRS, Skóra F, Abilhoa V. Homogenization of freshwater fish faunas after the elimination of a natural barrier by a dam in Neotropics. Divers Distrib. 2012; 18(2):111-20.

Ward JV, Stanford JA. The serial discontinuity concept of lotic ecosystems. In: Fontaine TD, Bartell SM, editors. Dynamics of lotic ecosystems. Michigan: Ann Arbor Science Publishers; 1983. p.29-42. 